Proceedings the 44th International School and Conference on the Physics of Semiconductors "Jaszowiec 2015", Wisła 2015

\title{
Correlative Optical Spectroscopy and Atom Probe Tomography
}

\author{
L. Rigutti* \\ Groupe de Physique des Matériaux, UMR 6634 CNRS, University and INSA of Rouen, Normandie University, \\ Avenue de l'Université BP12, St. Etienne du Rouvray, 76800, France
}

\begin{abstract}
This lecture will introduce and revise recent experimental developments on correlative laser-assisted atom probe tomography and optical spectroscopy, with a particular attention to the domain of semiconductor nanostructures. The main goal of correlative microscopy is to gain a deeper insight in materials science studies. For the materials scientist, indeed, the possibility of establishing a link between optical spectroscopic properties of a given system and the reconstruction of its 3D structure and composition by atom probe tomography yields an unprecedented insight into the complex influence of the structure on the electronic states and on the optical transitions characterizing the system. This lecture will therefore revise the different approaches by which it is possible to correlate optical spectroscopy experiments - in particular micro-photoluminescence with atom probe tomography and, possibly, with transmission electron microscopy. Dedicated sample preparation protocols and recent case studies will be reported. Finally, a perspective approach will be introduced, in which the same femtosecond laser pulse could be exploited not only for triggering ion evaporation, but also photon emission in situ in the atom probe itself.
\end{abstract}

DOI: 10.12693/APhysPolA.129.A-7

PACS: 61.46.- $-\mathrm{w}, 61.72 .-\mathrm{y}, 68.65 .-\mathrm{k}, 78.55 .-\mathrm{m}, 78.67 .-\mathrm{n}$

\section{Introduction}

Atom probe tomography (APT) is a technique allowing for the $3 \mathrm{D}$ reconstruction of the elemental composition of a nanoscale volume of a sharp (apex radius $\lesssim 100 \mathrm{~nm}$ ) specimen tip. It is based on the controlled field ion evaporation of the atoms at the tip surface and on their analysis through a position- and time-of-flight-sensitive detector, as schematized in Fig. 1a. The data accumulated during this controlled erosion of the specimen are collected and subsequently elaborated in order to reconstruct the $3 \mathrm{D}$ distribution of the constituent chemical species. It becomes then possible to reproduce sizes, shapes and chemical composition of nanoscale functional systems, accessing features that are beyond the reach of other nanoand atomic-scale instruments such as the transmission electron microscope (TEM) and the scanning tunneling microscope (STM) [1-3]. Since the 1990's, the control of field ion evaporation was carried out though voltage pulses, which was inadequate to the analysis of nonmetallic materials due to the propagation delays and to the bleaching of the voltage pulse towards the apex of the tip. The recent development of laser-assisted atom probe tomography (La-APT) [4] has made it possible to analyze a large variety of non-metallic nanostructures [5-7]. Among these structures, there are functional materials of particular interest because of their optical properties, such as the quantum dots shown in the 3D reconstructed volumes of Fig. 1b,c: for instance, because they can efficiently emit photons of a given energy and polarization, because they can emit single photons on demand, or because they can selectively absorb a certain interval of wavelengths. In many cases, the optical properties of nanoscale systems result from their structure and composition, which immediately makes it clear why it is interesting to analyze them by APT. The knowledge of the structure and composition of semiconductor quantum wells, quantum dots and nanocrystals or of metal nanoparticles at the nanoscale and in 3D opens the way to a complete understanding of their optical properties. This consideration is at the base of most of the present lecture, in which it will be dealt with the problem of connecting optical spectroscopic information with atom probe data at different levels of complexity and accuracy.

If the connection between optical properties and structural APT data is nowadays a reality with growing importance in the domain of nanosciences, there is another perspective interest in connecting optical spectroscopic information and La-APT, still requiring some instrumental development effort. In La-APT indeed the sample, a sharp tip containing a nanoscale functional structure is illuminated by a pulsed laser in order to trigger the ion evaporation. It is therefore possible to imagine that the same laser beam could be used in order to trigger photon emission and field ion evaporation. Similarly, the presence of specifically absorbing or scattering structures within the tip could leave a trace in the transmitted beam. The realization of an optical bench in situ inside an atom probe would open further possibilities for a more in-depth study not only of the properties of particular nanoscale structure, but also of the physical processes governing field ion evaporation itself. 
(a)

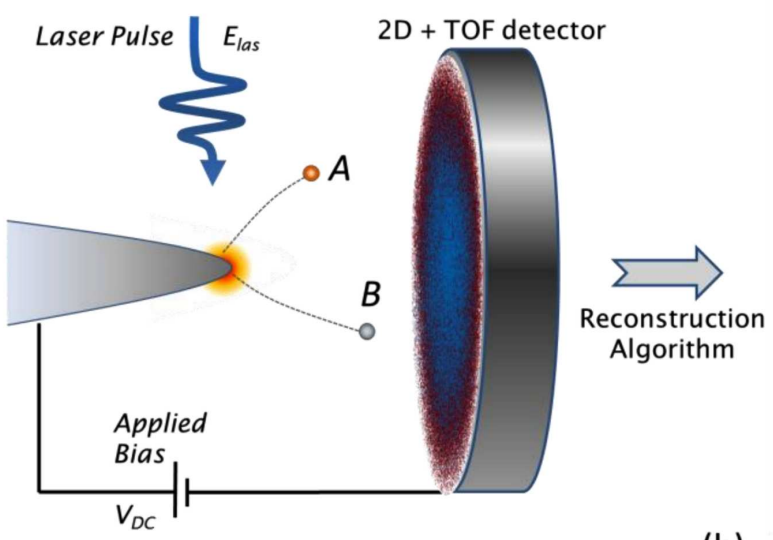

(b)

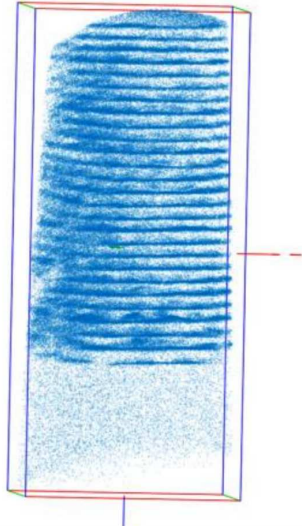

$20 \mathrm{~nm}$

(c)

Fig. 1. (a) Schematics of an Atom Probe Tomography experiment; (b,c) 3D reconstruction of a GaN/AlN StranskiKrastanov quantum dot system; notice that (b) and (c) constitute a stereogram, and may be looked at with the left and the right eye, respectively, in order to obtain a pleasant 3D impression.

\section{Relationships between chemistry, structure and optics in semiconductor nanostructures}

Under the expression "optical properties" of a given system there is included a large amount of information, relative to the interaction of the system with the electromagnetic field of light. This information may concern the fundamental electronic transitions giving rise to photon absorption, emission or scattering, but also the way the light couples to the system, for instance through waveguide or antenna effects. This lecture will deal with selected optical properties relative to light emission, mainly under photon excitation, i.e. what can be retrieved by performing photoluminescence (PL) or micro-photoluminescence ( $\mu \mathrm{PL})$ spectroscopy. It will also mainly deal with a specific subset of nanoscale systems, i.e. semiconductor heterostructures such as quantum wells and quantum dots.

In this above defined framework, optical emission spectroscopy can measure the following quantities relative to the emitted light:

\section{O1. Energy (wavelength);}

O2. Polarization;

O3. Intensity;

O4. Temporal evolution.

The study of the time evolution of the above quantities is possible in a time-resolved setup, based on pulsed excitation. The parametric dependence of spectral information on temperature, pressure, strain, applied electric/magnetic field, etc. is also possible, provided the experimental setup has been properly designed. This optical information may be related to the structural properties of the system, some of which are best accessed by APT:

S1. Size and shape of the emitting region;
S1. Chemical composition of the emitting region;

S3. Uniformity of the chemical composition (statistical alloy distribution, clustering, etc.);

S4. Presence of interfaces and their definition (abrupt or diffuse, smooth or rough, etc.);

S5. Presence of extended structural defects with a chemical trace (impurity segregation).

It is worth noticing that not all structural properties having an influence on the emission properties of a system are accessible by APT. Some of them are hardly, or not at all, accessible by APT:

S6. Presence of extended structural defects (stacking faults, dislocations) without a chemical trace;

S7. Presence of single impurities within specific regions;

S8. Intrinsic point defects such as interstitials or vacancies;

S9. Crystal symmetry and orientation ${ }^{\dagger}$;

S10. Strain state.

This shows that the combination of APT and optical spectroscopy may provide the researcher with an extremely rich amount of information about the interplay between structural and optical properties, but also that

\footnotetext{
†The crystalline symmetry and orientation could in principle be assessed by field ion microscopy (FIM) within an atom probe. Such studies are still quite rare for semiconductor tips and not always conclusive, which makes this an interesting domain of research to be developed in the next few years.
} 
this information might not be exhaustive, and that complementary analyses could be necessary in order to back up the conclusions.

In the following sections, the way in which structural properties determine optical properties will be made more explicit in the case of semiconductor heterostructures. The information provided here is necessarily succinct, but the provided references guide the reader towards specific and more detailed didactical literature.

\subsection{Bulk semiconductors}

In bulk semiconductors the fundamental optical transition in the NIR-Vis-UV range is the bandgap transition. Bulk semiconductors strongly absorb photons with energy higher than the bandgap, while the absorption of lower-energy photons is weaker. The absorption strength may vary from direct-bandgap semiconductors, in which the photon may be absorbed directly without creating or absorbing lattice vibrations (phonons) and which exhibit a stronger absorption, to indirect semiconductors, in which a phonon must be absorbed or emitted in order to have the photon absorbed. In this latter category of semiconductors (to which belong $\mathrm{Si}, \mathrm{Ge}, \mathrm{SiC}$ ) the absorption is a three-body process, which makes it less probable, and therefore weaker, than the two-body process of direct semiconductors. This also has consequences on the emission properties of the semiconductor: as a general rule, direct bandgap semiconductors can be used as active (emitting) elements in optoelectronics, while indirect semiconductors still may be used as passive elements (waveguides or absorbers). The dispersion relation (also called $E-k$ diagram) of a bulk direct semiconductor is schematically depicted in Fig. 2a. The conduction and valence bands are approximately represented as parabolas, while the distance between the top of the valence band and the bottom of the conduction band is the energy bandgap (or shortly, gap) $E_{\mathrm{g}}$. The bandgap is characteristic of the material, and depends on its chemical composition and on its crystal symmetry; this means, for instance, that polytypes with identical compositions but different crystal symmetry will have different bandgaps (e.g. SiC: $2 \mathrm{H}, 4 \mathrm{H}, 3 \mathrm{C}$ have $E_{\mathrm{g}}=3.33$, $3.265,2.39 \mathrm{eV}$, respectively [8]). It also means that the bandgap can be continuously tuned by controlling the chemical composition of binary, ternary or quaternary alloys (e.g. $\mathrm{Si}_{x} \mathrm{Ge}_{1-x}, \mathrm{In}_{x} \mathrm{Ga}_{1-x} \mathrm{As}, \mathrm{In}_{x} \mathrm{Ga}_{1-x-y} \mathrm{Al}_{y} \mathrm{As}$ ) during the synthesis of the material. The way how the gap of the $\operatorname{In}_{x} \mathrm{Ga}_{1-x} \mathrm{~N}$ alloy varies with the $x$ of $\mathrm{InN}$ alloy fraction is represented by the blue dashed line in Fig. $2 \mathrm{i}$ for $0<x<0.3$. In general, the dependence of the bandgap on the alloy fraction for alloys with binary components $\mathrm{A}$ and $\mathrm{B}$ (e.g. $\mathrm{Si}+\mathrm{Ge}$, or $\mathrm{InN}+\mathrm{GaN})$ can be described in the framework of the so-called virtual crystal approximation ( VCA) as a weighted average of the properties of the single components A and B (the Vegard law), corrected with a bowing parameter $b_{\mathrm{AB}}[9]$ :

$$
E_{\mathrm{g}}\left(\mathrm{A}_{x} \mathrm{~B}_{1-x}\right)=E_{\mathrm{g}}(\mathrm{A}) x+E_{\mathrm{g}}(\mathrm{B})(1-x)+b_{\mathrm{AB}} x(1-x) .
$$

The VCA holds for a large class of random alloys, but is not applicable to alloys exhibiting spontaneous ordering, and is also not adapted to the description of alloys such as dilute nitrides [10]. The relationship between the gap and the chemical composition becomes in these cases more complex.

On the other hand, the insertion of donor or acceptor impurities does not have an important effect on the bandgap of a semiconductor, unless the doping density attains particularly high values, giving rise to phenomena such as the Burstein-Moss shift due to the occupancy of a large quantity of available states in the bands by free carriers promoted from the impurities, or bandgap renormalization due to the self-energy of free carriers themselves. Such impurity densities depend on the properties of the host material [9].

In a semiconductor, the fundamental emission takes place across the bandgap. However, photons may have energies slightly differing from $E_{\mathrm{g}}$ because of many-body effects. Free electron-hole pairs, for instance, may bind together to hydrogen-like states called excitons, and then recombine radiatively producing photons with an energy $h \nu<E_{\mathrm{g}}[9]$.

The composition of a semiconductor alloy measured by APT is thus a first quantity that could be related to its optical emission or absorption properties.

Further emission mechanisms may arise if specific defects induce the presence of electron states within the gap (deep levels, at energy $E_{\mathrm{DL}}$ ). Some of these levels may yield radiative recombination. Examples for this behavior are the intrinsic defects giving rise to deep luminescence bands in GaN, or luminescent impurities, such as rare earth ions, which can be inserted into the host matrix during the synthesis [11]. This impurity concentration, if higher than $\approx 10^{18} \mathrm{~cm}^{-3}$, can be studied by APT.

\subsection{Heterostructured semiconductors and low-dimensional systems}

In semiconductor heterostructures, semiconductors with different bandgap are arranged in order to occupy different spatial regions. The primary goal of this approach is the so-called band engineering, i.e. the possibility of designing semiconductor systems with specific electronic properties, which constitute the functional building block of electronic and optoelectronic devices such as high electron mobility transistors, light-emitting diodes, laser diodes, single photon emitters or photodetectors. In many cases, such specific properties are obtained by confining the electron and/or hole states in extremely narrow planes (several $\mathrm{nm}$ ), cylinders or islands. This confinement makes wave-like properties of electrons in solids measurable, and is therefore a quantum phenomenon [12]. In the following, we will summarize the basic features of selected heterostructure systems and the influence of the structural properties accessible by APT on their optical emission properties. The reader interested in the physics of low dimensional systems can refer to several textbooks for further details [12-15]. 
(a)

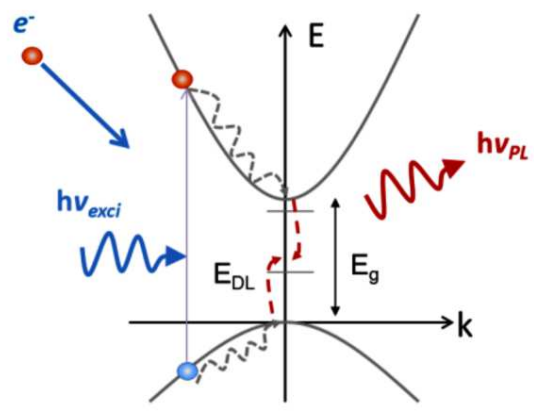

(b)
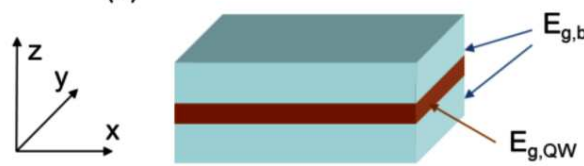

(d)

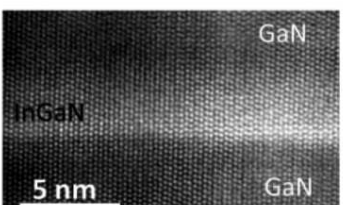

(c)

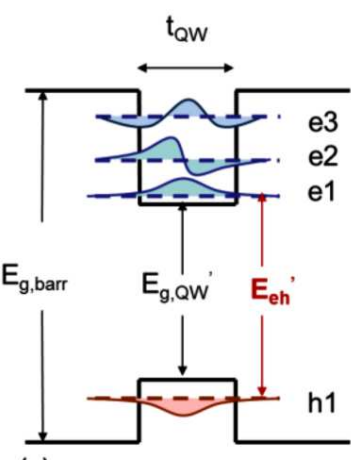

(g)

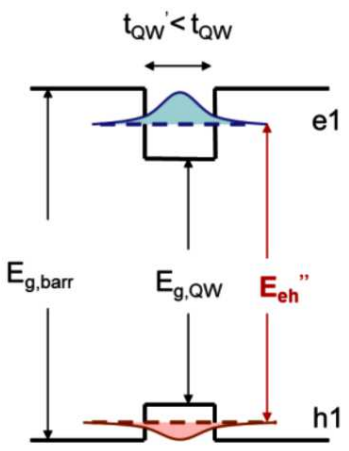

(h)
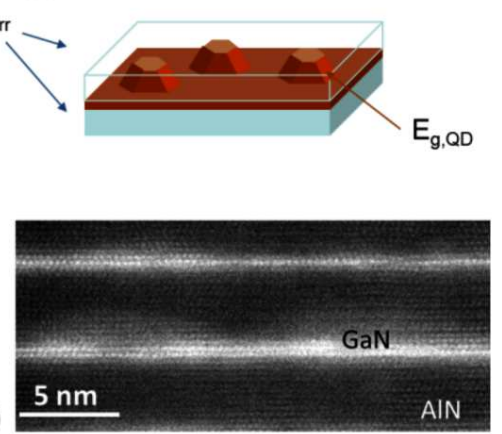

(e)

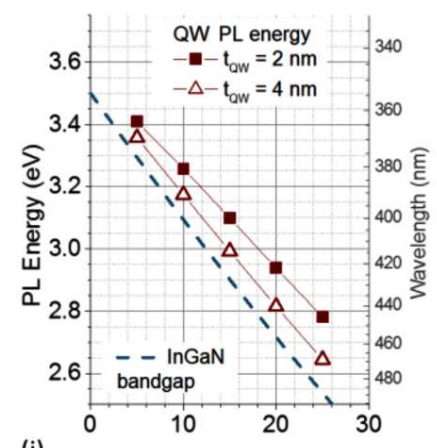

(i)

Fig. 2. (a) Scheme of electronic state excitation by photons or electrons with energy above the bandgap of a semiconductor, subsequent relaxation of electron (hole) to the bottom of the conduction (valence) band, and final radiative recombination of carriers yielding the emission of a photon with energy roughly equal to the bandgap. (b) Scheme and (c) HR-STEM image of a quantum well. (d) Scheme and (e) HR-STEM image of a Stranski-Krastanov quantum dot. (f) Potential profile and confined electron and hole energies and wave functions (sub-bands) within a rectangular quantum well of a given composition and thickness. Parts (g) and (h) show how the energy of the states and of the main radiative recombination evolve with quantum well composition and thickness, respectively. (i) Calculation of the recombination energy within an $\operatorname{In}_{x} \mathrm{Ga}_{1-x} \mathrm{~N} / \mathrm{GaN}$ quantum well defined on the $m$-plane of the wurtzite GaN crystal as a function of InN alloy fraction and quantum well thickness. The bulk InGaN bandgap is also traced for reference.

\subsubsection{Quantum wells}

In the simple picture, such as that represented in Fig. 2b, a quantum well (QW) is a thin flat layer (thickness $\left.t_{\mathrm{QW}}\right)$ of semiconductor with a gap $E_{\mathrm{g}, \mathrm{QW}}$ synthesized between two - generally thicker - layers of semiconductor with bandgap $E_{\mathrm{g} \text {,barr }}>E_{\mathrm{g}, \mathrm{QW}}$. An example of InGaN/GaN quantum well observed by high-resolution scanning electron transmission microscopy (HR-STEM) is also reported in Fig. 2c. If the bands of the different semiconductor phases align in such a way as that is reproduced in the scheme of Fig. $2 \mathrm{f}-\mathrm{h}$, the band profile across the quantum well is such that both electrons and holes can be confined within the well. Carriers in a quantum well are confined only in the growth direction (here denoted as $z$ ), but they can freely move along the quantum well plane $(x, y)$. They thus possess in-plane energy levels which depend on their in-plane wave vector, and are distributed in $2 \mathrm{D}$ bands. The total carrier envelope function - we neglect here the lattice periodic wave function - is the product between the confined wave function $\psi_{n(\mathrm{e}, \mathrm{h})}(z)$ and a plane wave $\exp (\mathrm{i} \boldsymbol{k} \cdot \boldsymbol{r})$ with $\boldsymbol{r}=(x, y)$. The Schrödinger equation of the system may thus be separately solved for the in-plane and the on-axis components. The on-axis solution yields a set of discrete levels, called subbands. The subband energy can first be estimated by approximating the barriers as infinitely high. In this ideal case, the energy $E_{n(\mathrm{e}, \mathrm{h})}$ of the electron/hole subbands can be indexed as follows [12]:

$$
E_{n(\mathrm{e}, \mathrm{h})}=\frac{\hbar^{2} \pi^{2} n^{2}}{m_{\mathrm{e}, \mathrm{h}}^{*} t_{\mathrm{QW}}^{2}}, \quad n=1,2,3, \ldots
$$

while the wave functions $\psi_{n(\mathrm{e}, \mathrm{h})}(z)$ are completely confined within the quantum well and are given by

$$
\psi_{n(\mathrm{e}, \mathrm{h})}(z)=A_{n} \sin \left(\frac{\pi n z}{t_{\mathrm{QW}}}\right) .
$$

Here, $m_{\mathrm{e}, \mathrm{h}}^{*}$ is the electron (hole) effective mass, which also depends on the QW composition ${ }^{\ddagger}$, while $A_{n}$ is the normalization constant satisfying the condition that the

¥A corresponding expression of the Vegard law may also be defined for the effective mass in alloys. We neglect here the possible anisotropy of the effective mass and the fact that more than one valence band may be involved in the determination of hole states. 
integral of the squared wave function over $z$ is equal to unity. One of the main effects of quantum confinement is that the electron and hole ground states do not lie at the bottom of the conduction or valence bands characteristic of the QW phase, but are lifted by a certain amount, which is called the confinement energy, $E_{1(\mathrm{e}, \mathrm{h})}$. Going back to a real quantum well, the finiteness of the barrier energy gaps yields an evanescent but nonzero wave function within the barriers and a decrease of the subband energy $E_{n}$, as depicted in the schemes of Fig. $2 \mathrm{f}-\mathrm{h}$. The wave function becomes thus progressively less confined with decrease of barrier gap. Furthermore, a finite number of subbands can be accommodated in a QW with a finite barrier height.

The main optical emission taking place within a quantum well involves the electron and hole ground states. As a first approximation, the emission energy is thus

$$
E_{\mathrm{e}, \mathrm{h}}=E_{1 \mathrm{e}}+E_{1 \mathrm{~h}}+E_{\mathrm{g}, \mathrm{QW}} \text {. }
$$

The terms appearing in this quantity all depend on the quantum well composition. The terms $E_{1 \mathrm{e}, \mathrm{h}}$ also depend on the barrier composition and on the quantum well thickness $t_{\mathrm{QW}}$. The way optical emission energies vary with varying well composition and thickness are schematically depicted in Fig. 2g,h: a decrease in the QW bandgap yields a decrease of $E_{\mathrm{e}, \mathrm{h}}$, while a diminution of the QW thickness yields its increase. This is also exemplified in the calculation reported in Fig. $2 \mathrm{i}$ in the case of non-polar ${ }^{\S} \mathrm{InGaN} / \mathrm{GaN}$ quantum wells.

Real quantum wells are of course more complex than the basic picture introduced here. The interfaces may be not properly defined, because of roughness or of alloy concentration gradients. Alloy clustering within the quantum wells may occur as well. Defects may be present as well. Some of these structural features may be properly addressed by atom probe, while for a complete understanding of the influence of all structural properties on photon emission a more complex approach is needed, involving for instance a complementary atomic-scale microscopy technique.

\subsubsection{Quantum dots}

Quantum dots (QDs) are islands of semiconductor with bandgap $E_{\mathrm{g}, \mathrm{QD}}$ embedded into a semiconducting matrix of larger bandgap $E_{\mathrm{g}, \text { barr }}>E_{\mathrm{g}, \mathrm{QD}}$. There are nowadays different methods for synthesizing such structures, such as the Stranski-Krastanov (SK) growth, droplet quantum dots, colloidal quantum dots, or quantum dots in nanowires. The scheme reported in Fig. 2d and the HRSTEM image of Fig. 2e illustrate the configuration of SK, whose strain-driven formation occurs on a wetting layer

\footnotetext{
§In polar crystals, heterostructure interfaces defined on polar planes yield a buildup of a static $2 \mathrm{D}$ charge density at the interfaces, due to pyroelectric and piezoelectric effects. These sheet charges induce large electric fields (order of several $\mathrm{V} / \mathrm{nm}$ in IIInitrides) which significantly affect the energy profile and the electronic states. See Sect. 2.2.3 for further information.
}

with the same composition of the dot deposited onto a flat substrate. The main difference between the quantum well and the quantum dot, is that in the dot the carrier wave functions are confined in the three dimensions. This no longer gives rise to energy quantization in one direction with the formation of subbands, but to the definition of discrete energy levels, analogously to those of isolated atoms. Even if there are important differences between individual atoms and quantum dots, the QD scheme allows for an easy manipulation of discrete and well-defined electron states, with important applications in the framework of single photon emission, quantum cryptography, and for fundamental quantum mechanics studies. As a first rough approximation, the energies and the envelope wave functions confined in a rectangular parallelepipedshaped QD embedded into an infinitely high barrier are given by

$$
\begin{aligned}
E_{\boldsymbol{n}(e, h)} & =\frac{\hbar^{2} \pi^{2}}{m_{\mathrm{e}, \mathrm{h}}^{*}}\left[\left(\frac{n_{x}}{t_{x}}\right)^{2}+\left(\frac{n_{y}}{t_{y}}\right)^{2}+\left(\frac{n_{z}}{t_{z}}\right)^{2}\right], \\
n_{x, y, z} & =1,2,3, \ldots
\end{aligned}
$$

and

$$
\psi_{\boldsymbol{n}(\mathrm{e}, \mathrm{h})}(\boldsymbol{r})=A_{\boldsymbol{n}} \sin \left(\frac{\pi n_{x} x}{t_{x}}\right) \sin \left(\frac{\pi n_{y} y}{t_{y}}\right) \sin \left(\frac{\pi n_{z} z}{t_{z}}\right)
$$

respectively. Here, $n_{x, y, z}$ are positive integers, while $t_{x, y, z}$ are the QD size in the each dimension. The possible anisotropy of the effective mass is not taken into account here, but could add a dependence on the crystal orientation and symmetry. In the case of real QDs, the finiteness of the barrier will influence the wave function and energies of the confined levels. Real quantum dots also do not generally have a parallelepiped shape. In the SK case, they rather have the shape of a truncated pyramid, while in nanowires they can be elaborated as cylindrical or also have more complex shapes due to alloy segregation and fluctuation effects. Furthermore, the composition of a QD could be non-uniform, as strain effects may lead to segregation of alloy components within the system. What should be underlined here is that both the 3D composition of the $\mathrm{QD} /$ barrier system and the $3 \mathrm{D}$ shape of the QDs determine the electronic and optical properties of a QD of a given crystalline symmetry. The interest of their study by APT resides therefore in the fact that only this technique currently allows for a reconstruction of their 3D shape and composition, and therefore for an in-depth understanding of the connection between optics and structure of the system.

\subsubsection{Quantum confined Stark effect}

Another interesting consideration which could relate APT and optical spectroscopy is the following: in semiconductor quantum confining systems, the PL emission energy as well as the absorption edge may be influenced by the presence of an electric field. This field could be (i) an internal field, such as in polar heterostructures, or (ii) an externally applied field. This is known as the quantum confined Stark effect (QCSE) [16], and it can 
be exploited in optoelectronic devices such as modulators and tunable absorbers [17].

Case (i) is illustrated in Fig. 3a,b, and may appear in semiconductors having crystal directions without inversion symmetry, such as the [0001] in the case of wurtzite crystals. The most studied system exhibiting polar properties leading to QCSE is the III-N system. If a spontaneous polarization develops and if this polarization is different for the different heterostructure phases, a static sheet charge builds up at heterointerfaces. This is a pyroelectric effect, and the sign of the charge depends on the polarization value in phases $\mathrm{A}$ and $\mathrm{B}$ constituting the heterointerface and whether phase A precedes or follows phase B along the polar direction. If, moreover, a heterostructure phase is coherently strained on another, the strain may set up a further polarization $\mathbb{I}$, leading to an increase in the absolute value of the sheet charge at the heterointerfaces - a piezoelectric effect. The buildup of sheet charges of opposite signs at heterointerfaces translates into the presence of an electric field within the quantum structure (or better, an electric field discontinuity across the heterointerfaces). In nitride quantum wells and dots, sheet charges may be of the order of $\approx 10^{-2} \mathrm{C} \mathrm{m}^{-2}$ and internal fields of the order of the $\mathrm{V} / \mathrm{nm}[18]$. As illustrated in Fig. 3a, the effect of the electric field is to reduce the electron and hole energies with respect to the flat-band case, and also to spatially separate the wave functions of electron and hole. This translates into modifications of the radiative recombination energy and intensity. Furthermore, the modification to the energy levels also depends on the thickness of the quantum confining structure. Figure 3b illustrates the dependence of the cathodoluminescence energy of GaN/AlN quantum discs ${ }^{\text {on }}$ their thickness. The experimental data show that the emission energy decreases well below the energy gap of GaN with increasing quantum disc: this would not be the case, as shown by the blue line - a calculation of the emission energies - if there was no field inside the well. The behavior of the experimental data indicates that a field discontinuity of around $0.4 \mathrm{~V} / \mathrm{nm}$ occurs between AlN barriers and GaN discs [12].

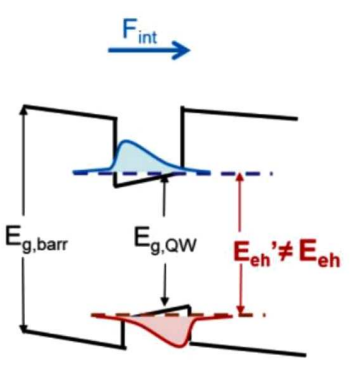

(a)

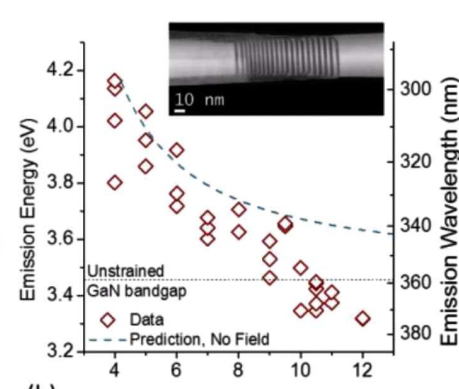

(b)
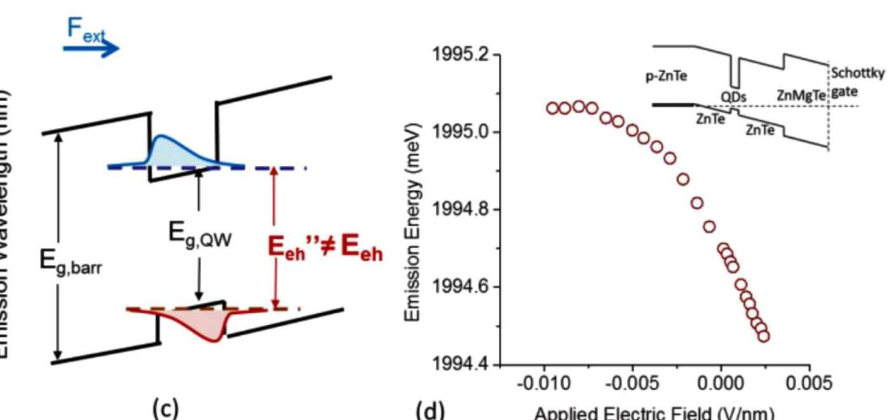

Fig. 3. (a) Potential profile and confined electron and hole energies and wave functions in a rectangular quantum well exhibiting the buildup of an internal field - the term $E_{\mathrm{e}, \mathrm{h}}$ refers to a zero-field system; (b) study of the quantum confined Stark effect in GaN/AlN quantum discs embedded in a nanowire and with hetero-interfaces defined on the polar (0001) planes - a STEM micrograph of the nanowire heterostructure is reported in the inset; the plot shows the dependence of the cathodoluminescence emission energies on the quantum disc monolayer (ML) thickness, with the blue line indicating the predicted values if no field was present (Adapted from [19]). (c) Potential profile and confined electron and hole energies and wave functions in a rectangular quantum well across which an external field is applied; (d) study of a CdSe/ZnSe quantum dot embedded in a Schottky junction - the scheme is reported in the inset; the plot shows dependence of the PL emission energy on the electric field applied to the junction (Adapted from [20]).

Case (ii) is illustrated in Fig. 3c,d. An electric field can be applied externally on a quantum confining system. Most frequently, this is done by embedding the

$\|$ Piezoelectric polarization may also set up in cubic crystals, as anisotropic strain may lift the inversion symmetry and modify the charge distribution so that a polarization builds up along a previously non-polar crystal direction.

$\|$ The quantum disc is a system with optical properties analogous to the quantum well. Unlike the quantum well, it is limited in $3 \mathrm{D}$, but with a higher aspect ratio between the radial and the axial size. Electron and hole states are not effectively quantumconfined along the radial direction, only along the axial direction, like in a QW. system in a $p-n$ or the Schottky junction, but QCSE induced by contactless techniques has been demonstrated as well [21]. In this way, it is possible to study the parametric dependence of the radiative transition energies and lifetime on the applied field. Figure 3c shows how the potential energy profile of a quantum well under an externally applied electric field looks like, while in Fig. 3d is shown the dependence of the PL energy of an excitonic emission measured in a CdSe/ZnTe QD embedded in a Schottky junction. It is interesting to notice that, as the dot is inside the depletion region of the junction, the built-in field of the junction is balanced when an externally applied bias produces an opposite field of around $-0.01 \mathrm{~V} / \mathrm{nm}$. 
Typically, quantum confining structures exhibit measurable Stark shifts with field values of the order of some fractions of $\mathrm{V} / \mathrm{nm}$. The magnitude of such fields should be compared with the surface field applied to an atom probe tip, which is of the order of some tens of $\mathrm{V} / \mathrm{nm}$ : if only a fraction of this high field would penetrate inside a field emission tip containing an optically active quantum emitting system, a significant Stark shift would be induced in its transition energies.

TABLE I

Experimental approaches for connecting optical properties and atom probe data of semiconductor nanostructures.

\begin{tabular}{|c|c|c|c|}
\hline $\begin{array}{l}\text { Experimental } \\
\text { approach }\end{array}$ & Description & Available information & Limitations \\
\hline $\begin{array}{l}\text { Statistically } \\
\text { correlative }\end{array}$ & $\begin{array}{l}\text { Optical spectroscopy and } \\
\text { APT performed on different } \\
\text { nanoscale parts of the same } \\
\text { macroscopic sample }\end{array}$ & $\begin{array}{l}\text { Optical ensemble properties, se- } \\
\text { lected structural properties of } \\
\text { nanoscale-sized samples. }\end{array}$ & $\begin{array}{l}\text { The connection between optics } \\
\text { and structure is statistical. }\end{array}$ \\
\hline $\begin{array}{l}\text { Strictly } \\
\text { correlative }\end{array}$ & $\begin{array}{l}\text { Optical spectroscopy and APT } \\
\text { performed subsequently on the } \\
\text { same nanoscale object }\end{array}$ & $\begin{array}{l}\text { Close correspondence between } \\
\text { optical and structural proper- } \\
\text { ties. } \\
\text { In the case of a single emitter, } \\
\text { exact correspondence. } \\
\text { Further correlation with HR- } \\
\text { TEM possible. }\end{array}$ & $\begin{array}{l}\text { Extremely careful sample } \\
\text { preparation needed, high risk of } \\
\text { failure due to multiple sample } \\
\text { manipulation. } \\
\text { Possible negative impact of FIB } \\
\text { on optical properties. } \\
\text { Correspondence lost if optical } \\
\text { emitter is outside the APT field } \\
\text { of view. }\end{array}$ \\
\hline Coupled, in situ & $\begin{array}{l}\text { Optical spectroscopy and } \\
\text { APT performed on the same } \\
\text { nanoscale sample in situ within } \\
\text { the atom probe }\end{array}$ & $\begin{array}{l}\text { Compact correlative approach, } \\
\text { minimization of manipulation- } \\
\text { related specimen fracture. } \\
\text { Possible spectral profiling dur- } \\
\text { ing tip erosion. Exact corre- } \\
\text { spondence between optical and } \\
\text { structural properties even for } \\
\text { multiple emitters within a sin- } \\
\text { gle sample. } \\
\text { Study of effect of the applied } \\
\text { DC electric field on the optical } \\
\text { properties. }\end{array}$ & $\begin{array}{l}\text { Same limitations as in the cor- } \\
\text { related approach. } \\
\text { Need of matching sample tem- } \\
\text { perature, applied field and inci- } \\
\text { dent laser energy in order to si- } \\
\text { multaneously perform APT and } \\
\text { optical spectroscopy. } \\
\text { Further correspondence with } \\
\text { HR-TEM not possible if optical } \\
\text { properties need to be preserved. }\end{array}$ \\
\hline
\end{tabular}

\section{Experimental approaches}

The experimental connection between optical spectroscopy and atom probe can be established by different means, depending on the available experimental facilities, on the desired degree of accuracy and on the system under study. In this chapter, the experimental approaches have been classified as follows:

- Statistically correlative approaches, in which different (possibly nanoscale) subsystems of a given macroscopic system (a thin film, or a nanowire array, for instance) are studied separately by optical spectroscopy and atom probe tomography. This approach has become quite common in semiconductors nanosciences, as it does not present any major blocking point from the experimental point of view.

- Strictly correlative approaches, in which the same nanoscale system is studied before by optical spectroscopy, and after by APT. This approach is quite challenging, as it is not obvious to prepare an atom probe sample (or a system with size close to an atom probe sample) preserving its optical properties. Also, not any optically interesting nanoscale system is adapted to a correlated study.

- Coupled, in situ approach, in which an optical spectroscopy bench is implemented in an atom probe instrument, allowing for in situ optical study within the atom probe itself. This approach has not been demonstrated yet, but its feasibility is under study.

The main features, advantages and drawbacks of these three classes of approach are summarized in Table I and will be discussed in the following sections, reporting and explaining selected examples issued from recent studies.

\subsection{The statistically correlative approach}

By a statistically correlative study is meant a set of experiments performed by optical spectroscopy and APT on different parts of the same macroscopic sample. In practice, this could consist for instance in studying the macroscopic PL from a large surface of a thin film sample and in the extraction of a field emission tip to study by APT, or, as another example, in the study of a given 
set of ensemble or individual nanowires by optical spectroscopy, and in parallel by the APT study of another set of nanowires. Thus, the comparative experiment relies on the assumption that the sub-systems studied by optical spectroscopy and APT are statistically representative of the overall properties of the macroscopic system studied. This does not mean that it is not possible to draw conclusions on the microscopic causes of the optical properties under analysis, only that the conclusions will be statistical in nature, and that a more complex approach is needed in order to correlate the structural properties of a given microscopic system imaged by APT and its own, individual optical properties. However, the statistically correlative approach has already proven to be very powerful for understanding the influence of structure on the optical properties of a larger and larger class of systems. In the following, three instructive examples of optical and APT studies will be illustrated in more detail.

3.1.1. GaAs/AlGaAs nanowire heterostructures and quantum dots

Another example of comparative optical-structural study by $\mu \mathrm{PL}$ and APT is relative to core-multishell GaAs-AlGaAs nanowires. The structure of such a nanowire is reported in Fig. 4a. These structures exhibit narrow line and single photon emission at energies higher than the GaAs bandgap, as shown in the spectrum and in the peak histogram of Fig. 4d,e, indicating that quantum dots may have formed within the AlGaAs shells.
The AlGaAs shells are not uniform indeed, as shown in the cross-sectional TEM image of Fig. 4b. Segregation of

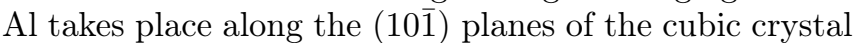
(the nanowire axis is oriented along the [411] direction) at the vertices of the hexagons defining the shell boundaries. It was also found by TEM that at the outer edge of the $\mathrm{Al}$ segregation region, nanometer-sized Al-poor regions may form, with facets defined on the $(1 \overline{1} \overline{2}),(\overline{2} 11)$, and (121) planes. These regions may confine electrons and holes, and can be therefore considered as quantum dots [22]. However, TEM could not indicate how deep these regions extend over the [4111] axis, and how the axial interfaces are defined.

These structures were therefore analyzed by APT, in order to verify the information about the QDs found by TEM and to further investigate the alloy distribution in the shell regions outside the segregation planes. A total of 5 wires, with an average depth of analysis of $400 \mathrm{~nm} /$ wire were studied by APT. As the wire section is quite large, and focused ion beam (FIB) is necessary in order to obtain sufficiently sharp tips, the imaged wire section is much smaller than the whole wire cross-section; however, it is still possible to image correctly the AlGaAs shell, including one or two Al segregation planes. Such a reconstructed volume is shown in Fig. 4c. The isosurfaces at $8 \%$ and at $42 \%$ AlAs fraction threshold, in red and blue, indicate the boundaries of the AlGAs shell and of the segregation planes, respectively. (a)

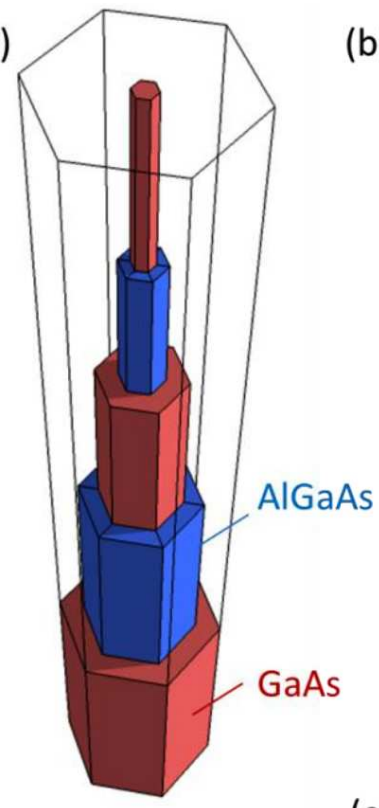

(c)

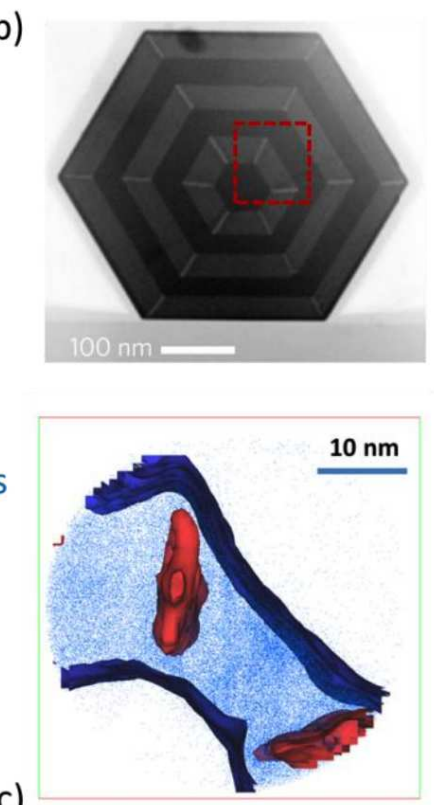

(d)
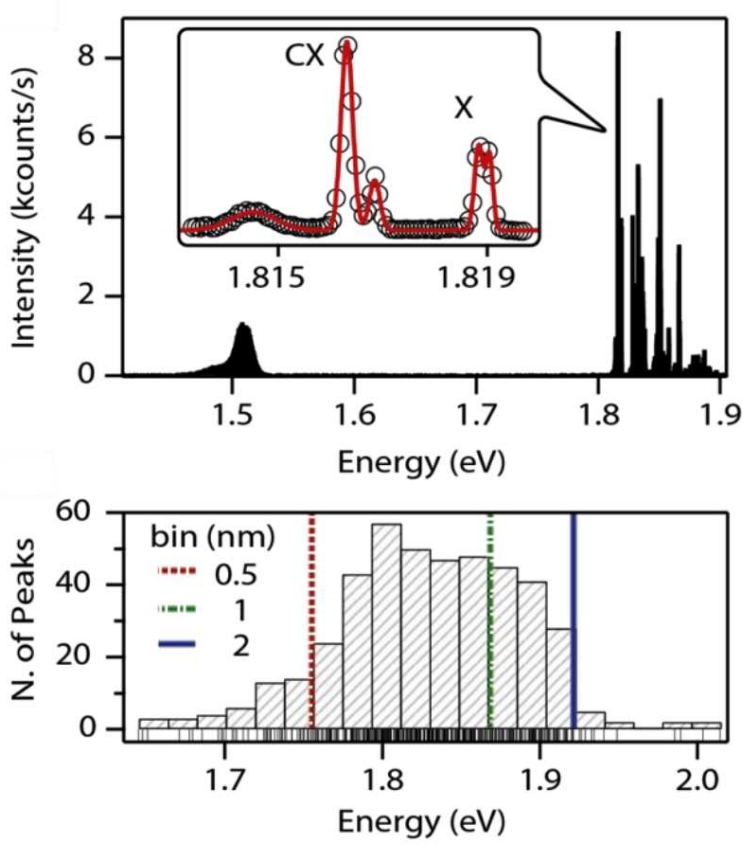

Fig. 4. (a) Scheme of the core-multishell GaAs/AlGaAs nanowire structure; (b) STEM-HAADF image of the crosssection of a nanowire: the AlGaAs shells exhibit Al segregation along the (101) planes connecting the vertices of the hexagons, visible as bright contrast lines; (c) APT 3D reconstruction of the fraction of nanowire volume: $\mathrm{Al}$ atoms are displayed in blue, while the blue (red) isosurface refers to $8 \%$ (42\%) AlAs alloy fraction. (d) Sample $\mu$ PL spectrum from a nanowire, exhibiting a GaAs-related emission at $1.5 \mathrm{eV}$ and multiple narrow line emission at higher energy, related to the formation of QDs in the AlGaAs shells; (e) statistical distribution of the narrow line emission energies issued by a large ensemble of nanowire spectra. The energies calculated for QD transitions based on the alloy density extracted from APT data for different volume bin size are superimposed (reproduced with permission from [23]). 
(a)

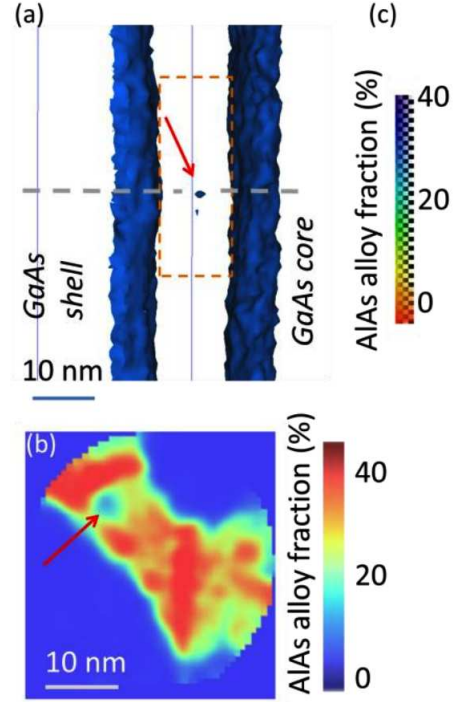

(c)

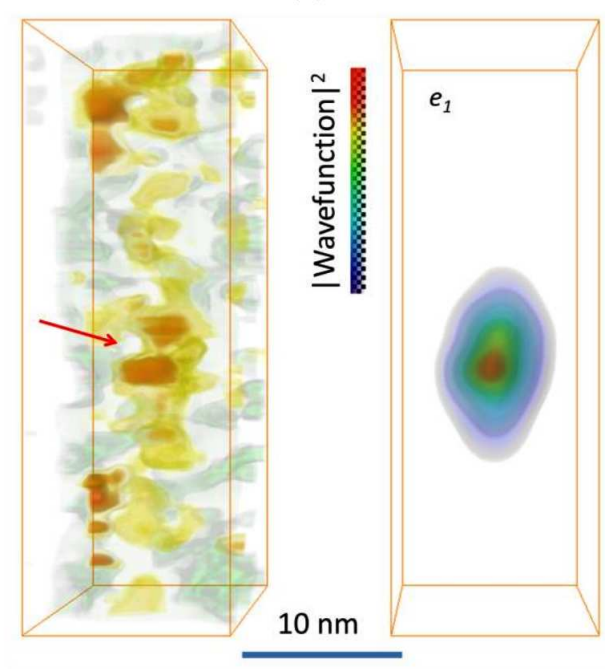

(e)

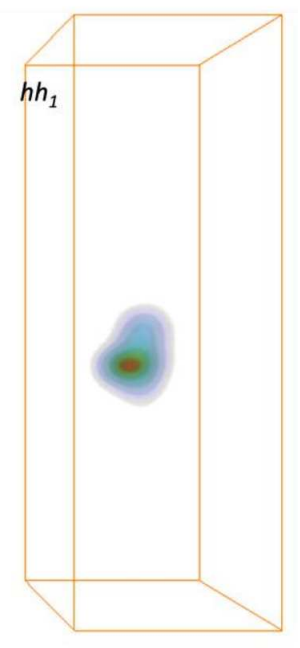

Fig. 5. (a) Atom probe 3D reconstruction of the sub-volume of sample NW3 containing a QD in the AlGaAs shell, showing the isosurfaces at $14 \%$ AlAs alloy fraction; the GaAs core and the external GaAs shell appear on the right and on the left-hand side, respectively; (b) 2D map of Al elemental concentration, extracted from the $1 \mathrm{~nm}$ thick slice corresponding to the grey dashed line in (a). The red arrows point to the QD position. (c) 3D visualization of the AlAs alloy fraction distribution in the region of interest highlighted in (a). (The low $\mathrm{Al}$ density regions on the edges correspond to the superposition of the ROI with the GaAs shell.) (d) and (e) Wavefunctions of the single-particle ground states of electron (d) and heavy hole (e) calculated in the framework of an effective mass approximation for the alloy distribution in (c) (reproduced with permission from [23]).

In the present set of studies, no evidence for QDs at the position and with the shape found in Ref. [22] was established. However, it appeared quite clearly that the alloy distribution out of the segregation planes is not uniform, and that strong fluctuations may take place, as visualized in Fig. 5a-c. Such fluctuations may be so strong to yield efficient electron and hole confinement. Figure $5 \mathrm{c}$ reports the AlAs fraction map issued from APT data. The red arrow points to a strongly Al-poor region, or, in other words, to a quantum dot defined by an AlAs concentration gradient within the AlGaAs shell. Such a feature is extremely difficult to reveal by other means than APT, but has all features of a QD, as it can efficiently confine electron and hole levels in 3D. In order to verify the found QD could be compatible with the spectral properties as measured by PL, the AlAs fraction map was fed into the Schrödinger-Poisson solver Nextnano [24]. The calculated wave function shapes for electrons and heavy holes, calculated in the framework of an effective mass approximation, are shown in Fig. 5d,e. It is also noteworthy that the recombination energy is compatible with the peak histogram reported in Fig. 4e. However, it must be underlined that due to the extremely small size of the dot, the definition of the alloy fraction map and, consequently, the calculation of the $\mathrm{e}-\mathrm{h}$ wave functions and energies are very sensitive to the volume binning size - cubic binning volumes were adopted here and smoothing. In the shown calculations, no smooth- ing or delocalization were performed on the atom impact positions, as this would artificially decrease the confinement and increase the emission energy to values close to the average bandgap of the AlGaAs matrix. For the same reason, no binning size larger than $2 \mathrm{~nm}$ (i.e. about one half of the dot width) was considered. On the other hand, binning sizes smaller than $0.5 \mathrm{~nm}$ were not statistically sound, and were not considered either. This example shows therefore not only the advantages of the comparative PL and APT study, but also its limits: for extremely small objects such as the QD above, the uncertainties related to the spatial resolution of APT and to the statistical analysis of atom counts in small volumes translate into a large uncertainty of the predictable optical properties.

\subsection{The strictly correlative approach}

A strictly correlative experiment at the nanoscale consists in the study with different techniques of the same nanoscale object, or of a set of nanoscale objects, each of which is addressed individually by the two techniques. The gain in information that can be achieved by this approach is great: the link between the optical and structural information that can be achieved by comparative approaches is statistical in nature, while a correlative approach establishes individual links at the nano-object level. This is why a lot of different strictly correlative methods are flourishing in nanosciences, and this is why 
nowadays there is an increasing number of studies reporting on the correlation between optical emission [25] or optical extinction properties [26] and transmission electron microscopy. However, if one considers correlating an optical study with APT, one becomes rapidly aware that the task is not as straightforward as for a correlation with TEM.

The main reasons for this are the following: on the one hand, it is difficult to study a given isolated nano-object, subsequently transfer it onto a support tip and have it analyzed by atom probe; on the other hand, it is not obvious to prepare a nanoscale specimen on a support tip that could be studied by optical spectroscopy before, and by APT after. The first difficulty is mainly related to nanomanipulation issues, the second one to the use of FIB and its heavy impact on the optical properties of nanoscale objects.

\subsubsection{The FIB bottleneck}

The main obstacle to the implementation of a correlated approach is related to the use of FIB for the preparation of atom probe semiconducting and dielectric specimens. The preparation techniques based on FIB have been extensively treated in chapter FIB and the reader can refer to it for further detail. Figure 6 illustrates how FIB can affect the optical properties of a given sample. The first important consideration is that any exposure of a specimen to FIB, at any typical ion energy, produces some extent of implantation. Figure $6 \mathrm{a}$ shows the example of the trajectories of $\mathrm{Ga}$ ion of dif- ferent energy impinging at normal incidence onto a GaN volume, as simulated by SRIM [27]. Ions with energy $\approx 2 \mathrm{keV}$ are implanted on a relatively reduced thickness of several nm, this thickness significantly increases to over $10 \mathrm{~nm}$ for $30 \mathrm{keV}$ ions, which is the typical energy used for specimen milling operations. Figure $6 \mathrm{~b}, \mathrm{c}$ illustrates how the ion doses used for the preparation of Si slices, which do not differ too much from those used in APT specimen preparation, induce the amorphization of an external layer, whose thickness is around $2 \mathrm{~nm}$ for $2 \mathrm{keV}$ ions and around $20 \mathrm{~nm}$ for $30 \mathrm{keV}$ ions [28]. This latter value is already quite close to the size of an APT tip specimen. Moreover, ion implantation can take place even further than the thickness of the amorphized region, producing structural damage, extended and point defects. The effect of this on the radiative recombination properties of a sample are illustrated in Fig. $6 \mathrm{~d}$ in the case of a uniformly crystalline sample. As the crystalline properties of the sample are preserved far from the surface, generation of electron-hole pairs is still possible upon absorption of a photon with energy higher than the bandgap. However, the created carriers can easily diffuse towards the damaged surfaces, where their nonradiative lifetime $\tau_{n r}$ dramatically decreases because of the high density of nonradiative recombination centers. The radiative lifetime $\tau_{r}$ is unaffected with respect to the bulk material, so the relationship $\tau_{n r} \ll \tau_{r}$ translates into a very efficient decrease of luminescence efficiency.
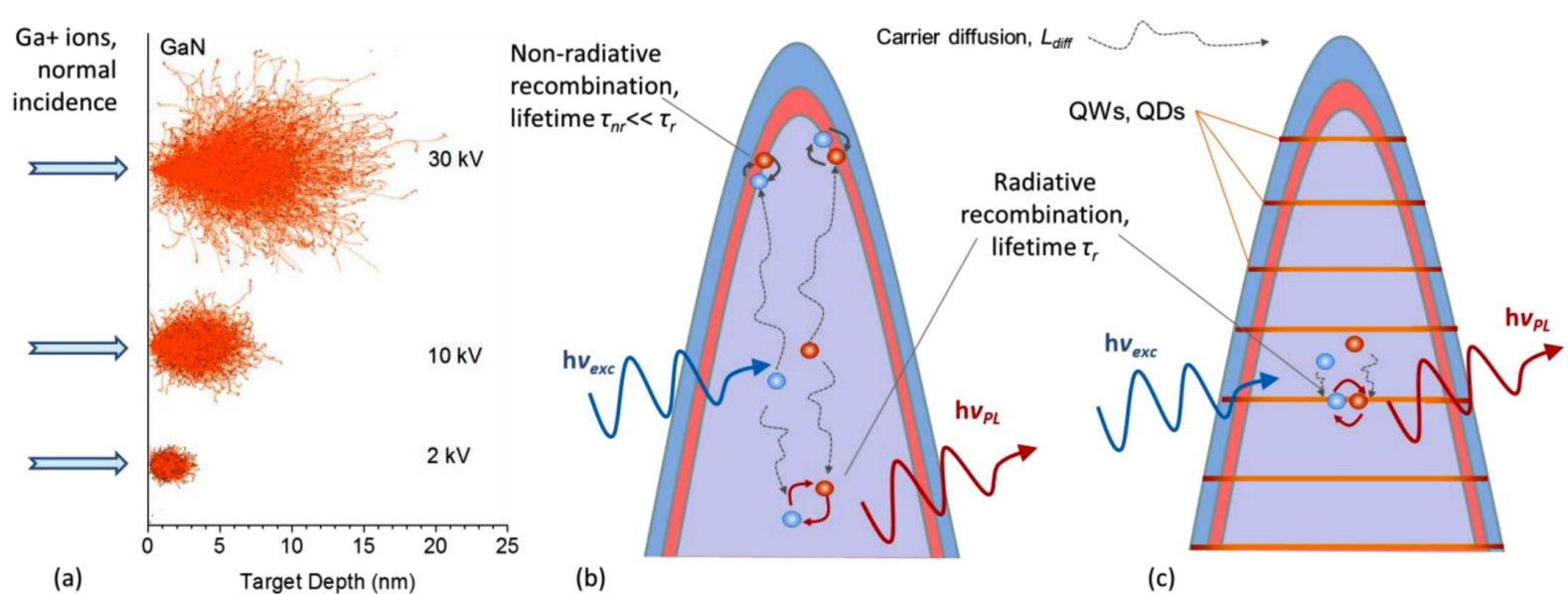

(b)

(c)

Fig. 6. (a) Trajectories of Ga ions impinging at normal incidence on GaN and at different energies, as given by SRIM calculation [27]. (b,c) Schematics of carrier generation, radiative and non-radiative recombination processes in a tip sample having a damaged surface: (b) represents a homogeneous tip, (c) a tip including quantum confining structures.

Before presenting with concrete data how the FIBinduced degradation of the radiative efficiency affects real samples, we make two important considerations meant to guide the operator. The first one is that independently of the implantation depth - and therefore of the ion energy - the presence of a damaged surface is sufficient to increase the nonradiative recombination rate.
Decreasing the ion energy may thus limit the damage, but would never totally resolve the problem of surface degradation of optical properties. The second consideration is that the effect of FIB is strong because APT specimen tips have a large surface-to-volume ratio: the surface is always very close to the region where carriers are generated, and diffusion towards the surfaces is an 
important process for the enhancement of nonradiative efficiency. So, if a sample contains some structure capable of reducing the diffusion length, this would help prevent the carriers from reaching the surface. Furthermore, if the diffusion-limiting structure also could locally enhance the radiative recombination efficiency, the effect of FIBinduced damage would not have a strong impact. This situation is depicted in the scheme of Fig. 6e. In this case, the presence of a multi-QW system is meant to both limit the carrier diffusion [29] and to increase their recombination probability once the carriers are captured by the quantum wells. Another possibility, not shown here, is the so-called resonant excitation, consisting in creating carriers directly within quantum confining subsystems (quantum wells or dots), from which they could not freely diffuse. In this case, the incident light should have well-determined photon energy, between the e-h1 transition energy and the barrier energy bandgap.

(a)

(b)
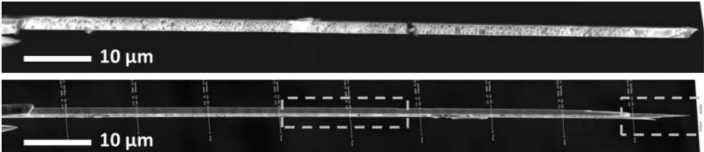

(c)

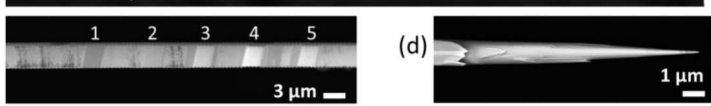

(e)
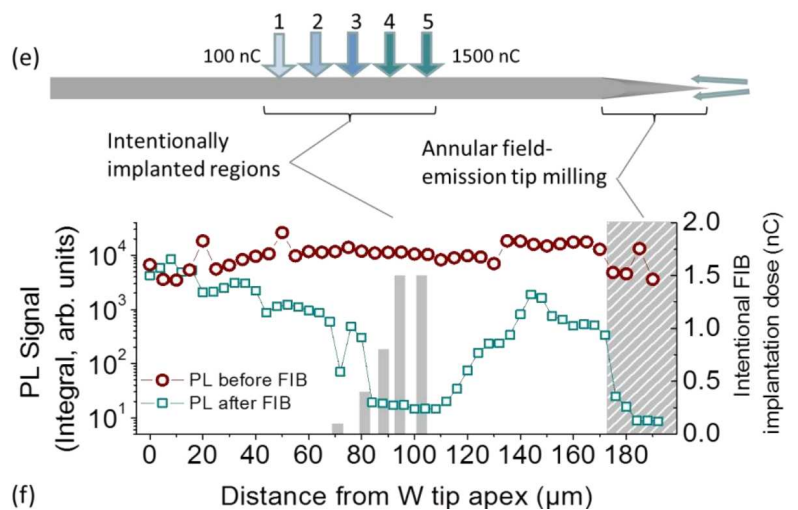

Fig. 7. (a) SEM image of a long $\mathrm{ZnO}$ microwire prepared for testing the effect of FIB on the optical properties of nanoscale systems; (b) the same nanowire after the intentional exposure of selected squared regions to different doses of $30 \mathrm{kV}$ Ga ions (zoom in (c)) and after annular milling of a field emission tip for atom probe analysis by $30 \mathrm{keV}$ ions and final $2 \mathrm{keV}$ cleaning step (zoom in (d)); (e) Spatially-resolved profile of the integrated micro-PL signal along the wire before and after FIB treatment, showing a strong modification of the optical properties due to the exposure to $30 \mathrm{keV} \mathrm{Ga}$ ions, particularly in the intentionally exposed regions (adapted with permission from [30]).

A study of the effect of FIB on the radiative recombination properties of $\mathrm{ZnO}$ is displayed in Fig. 7. In this study, a $\mathrm{ZnO}$ microwire $(200 \mu \mathrm{m}$ long, $4 \mu \mathrm{m}$ diameter) was used in order to test its resistance with respect to both intentional and unintentional exposure to $30 \mathrm{kV}$ ions. The wire was mounted by micromanipulation onto a $\mathrm{W}$ support tip, as shown in Fig. 7a and analyzed by spatially-resolved $\mu \mathrm{PL}$ at room temperature (i.e. scanning with the laser spot along the wire). The $\mu \mathrm{PL}$ spectra exhibited a quite uniform shape (not shown) and integral intensity along the wire, as reported in the profile of Fig. 7f. After that, the wire was treated by FIB. A field emission tip was annularly milled at the free extremity, while selected squared regions of $4 \times 4 \mathrm{\mu m}^{2}$ size were exposed to well-defined ion doses, ranging from 100 to $1500 \mathrm{nC}$, as shown in the SEM images and in the scheme of Fig. 7b-e. However, the entire wire surface on the FIB gun side was also unintentionally exposed to the ion beam due to imaging during wire orientation, with doses which are difficult to estimate. The $\mu \mathrm{PL}$ analysis after FIB exposure, performed under the same experimental conditions as previously specified, showed that the PL signal is lost - dropping below the noise level - in all intentionally exposed regions, with the exception of the region implanted with $100 \mathrm{nC}$ : here the signal drops by "only" almost two orders of magnitude but is still measurable. Also the unintentionally exposed regions exhibit a significant decrease of radiative recombination efficiency, which clearly indicates that the damage produced by FIB in uniform nanoscale systems induces a dramatic degradation of the radiative recombination properties. For completeness sake, a small volume of the field emission tip obtained from this long $\mathrm{ZnO}$ microwire was also analyzed at $80 \mathrm{~K}$ in an atom probe [30].

\subsubsection{Soft-FIB preparation protocols}

This section is dedicated to adapted FIB-based protocols meant to preserve the optical properties of systems for correlated optical and atom probe analysis. Basically, the protocol consists in a two-step FIB preparation, described in Fig. 8. The limit of this approach is that the two-step method prepares a structure for optical study which is slightly to significantly larger than the final field emission tip, weakening the correlation as the volumes studied in the two phases are not the same. The example reported in this figure is relative to micro-wires, but is can be easily adapted to thin film or bulk samples. The micro-wires studied were lying horizontally on a flat substrate, and were preliminary coated on their upper surface with a $400 \mathrm{~nm}$ thick Si layer, deposited by ion beam physical vapor deposition. This constitutes a protective layer preventing the FIB $30 \mathrm{keV} \mathrm{Ga+} \mathrm{ions}$ from penetrating inside the active region throughout the preparation. Subsequently, as shown in the scheme and in the SEM image of Fig. 8a, a section of a chosen microwire is welded by FIB-assisted $\mathrm{Pt}-\mathrm{C}$ deposition on the flat tip of a tungsten filament inserted in an aluminum capillary. The orientation of the wire section with respect to the capillary may be recorded for retrieving crystallographic information in the subsequent experiments if needed. Then, FIB annular milling is performed in two steps. In a first step, shown in Fig. 8b, the volume of the wire section is reduced to a small cylinder whose diameter is of the order of $500-200 \mathrm{~nm}$. The nanoobject obtained, to which we refer to as "nanochunk", is 
then ready for the $\mu \mathrm{PL}$ experiment. The relationship between PL intensity per unit volume and nanochunk diameter reported in the plot of Fig. 8d relative to a 20 -fold InGaN/GaN QW system indicates that diameters down to $200 \mathrm{~nm}$ are large enough to preserve the optical properties of the multi-QW system. Data for smaller diameters are still missing, but this study could be performed in the future: it would be desirable, indeed, to study systems for which this two-step protocol could be reduced to a single-step protocol. After performing $\mu \mathrm{PL}$ spectroscopy, a second and final annular milling step is carried out in order to obtain a sufficiently thin tip for analysis in the atom probe (apex radius lower than $50-75 \mathrm{~nm}$ ). The obtained field-emission tip is visualized in Fig. 8c. Note that this tip is also transparent to electron illumination and can be therefore analyzed in the HR-STEM before being eroded by APT. (a) As- mounted chunk

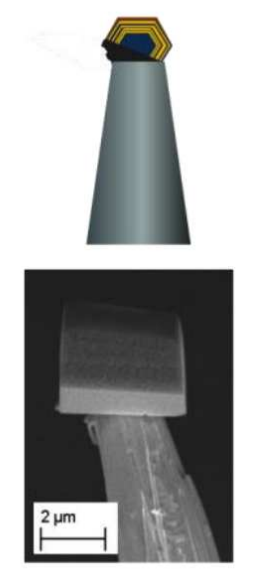

(b) Nano-chunk

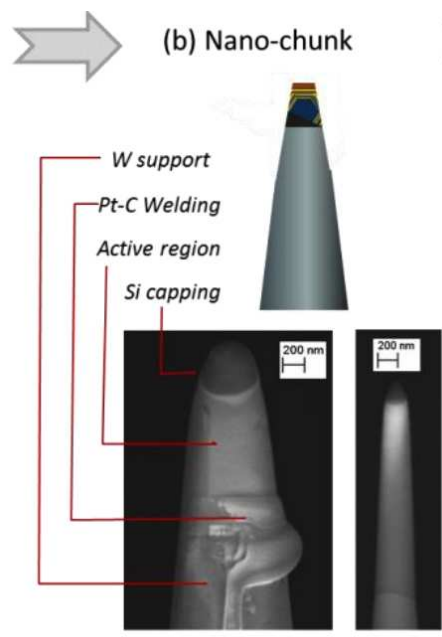

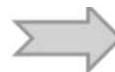

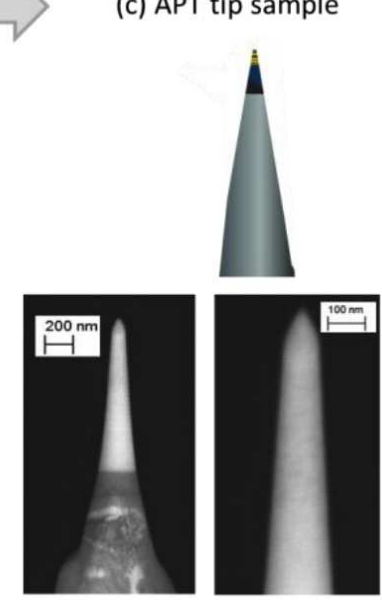

(d)

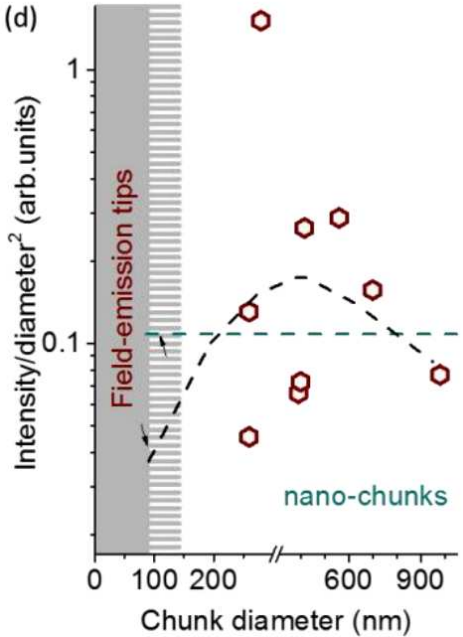

Fig. 8. Adaptive FIB protocol for correlated studies of an InGaN/GaN multi-QW system in a microwire: (a) A p-sized chunk is mounted and soldered onto a W support tip; (b) the chunk is annularly milled to an intermediate diameter approximating that of an atom probe sample tip (nanochunk): this volume can be studied by $\mu \mathrm{PL}-$ the SEM images refer to two different structures; (c) the nanochunk is finally milled down to the size of an atom probe sample tip and can be studied by APT - the SEM images refer to the same structure, on which a preliminary TEM study is also feasible. (d) PL signal per unit volume extracted from nanochunks as a function of their diameter.

\subsubsection{Strategies to limit the impact of FIB}

As a conclusion of this section, some recommendations are given for an implementation of FIB protocols as respectful as possible of the optical properties of the samples under study:

- Expected ion damage. The operator should keep in mind what is the penetration depth of the FIB in the material under study; such information can quite accurately be retrieved through SRIM simulation. Systems in which the optically active regions are isolated and sufficiently far from the surfaces should prove more resistant than homogeneous materials.

- Capping. The optically active region of the system should be protected as long as possible during the sample milling, for instance though preliminary capping with a passive metal layer.

- Soft finalization. The final steps of ion milling should be performed with low ion energies (below $10 \mathrm{kV}$ ). This will not avoid the presence of a damaged surface layer, but it will limit is impact as much as possible. However, the spatial accuracy of annular milling decreases with decreasing ion energy (the beam becomes less focused and the tip shape develops a larger cone angle) so the operator should find the good compromise between sufficient spatial accuracy and reduced ion penetration.

\subsubsection{FIB-free preparation protocols (nanowires)}

If FIB is detrimental to a correlated optical and APT study, specific structures exist for which the use of FIB is not required in order to prepare an atom probe specimen. Metals, for instance, can be electropolished to acquire the size of a field emission tip, and they also may produce photoluminescence, possibly assisted by plasmon-related absorption of exciting light $[31,32]$. This is a very interesting domain, which is surely worth studying, but which will not be dealt with here. Another class of materials is semiconductor nanowires: these structures can be indeed synthesized with large length/diameter aspect ratio, can contain heterostructures and quantum confining structures, and are being extensively studied because of their peculiar optical properties [33]. Provided the nanowire diameter is sufficiently small, of the order of $100 \mathrm{~nm}$ or 
smaller, nanowires can be mounted on support tips and be analyzed by $\mu \mathrm{PL}$ or other optical spectroscopy and subsequently by APT.

FIB-free protocols for the preparation of nanowires for correlated optical-APT studies can be various, but usually consist of three main steps:

- Nanowire pre-positioning;

- Nanowire collection and positioning on a support tip;

- Nanowire soldering.

By nanowire pre-positioning are meant all preliminary operations by which nanowires are separated by their original growth substrate and transferred into suitable positions, from which it becomes convenient to collect them by micromanipulation under an optical or scanning electron microscope, as schematized in Fig. 9a. This step is mandatory if nanowires on their growth substrate are too dense, or if they grow in directions making it difficult collect them with a support tip. In these cases, nanowires can be transferred onto another substrate or onto the edge of another substrate so that they are dispersed with a convenient density and orientation to pick them up later. Such a transfer can take place by ultrasound bath of nanowires on their growth substrate, their dispersion in a solvent, and the deposition of a solvent droplet on a host substrate, or by mechanical transfer, i.e. by simply scratching the growth substrate with a host substrate. In the latter case, the control on the NW density is much worse, but density gradients often occur in this random approach, so it is often possible to come across a region with a sufficient number of nanowire candidates for further processing.

Nanowire collection may occur with different approaches and configurations, essentially depending on the nanowire size and on whether it can stick to the support tip, which could be the final tip for APT analysis or an intermediate support from which the nanowire will be transferred on the final tip: for sake of simplicity, we assume that this support is the final one, as depicted in Fig. 9b. Collection may be carried out under an optical microscope, provided the nanowires are visible: this implies, as a rule of thumb, a nanowire length above $2 \mu \mathrm{m}$ and a diameter over $50 \mathrm{~nm}$. Otherwise, the wire still can be collected in an electron microscope, provided the SEM is equipped with suitable micromanipulation tools. The support tips may have been preliminarily shaped by FIB in order to assure that the nanowire is collected with its axis along the axis of analysis of the APT.

After or during collection, nanowire soldering should be carried out, in order to efficiently dissipate the heat accumulated during illumination by the lasers employed in optical spectroscopy or in APT. Soldering is also necessary to assure a good conduction between specimen tip and support tip and to mechanically hold the specimen tip at place during the APT run. For these reasons, the soldering should be rather conductive than insulating. For nanowires, there are several alternatives. Micromanipulation under optical microscope could be assisted by

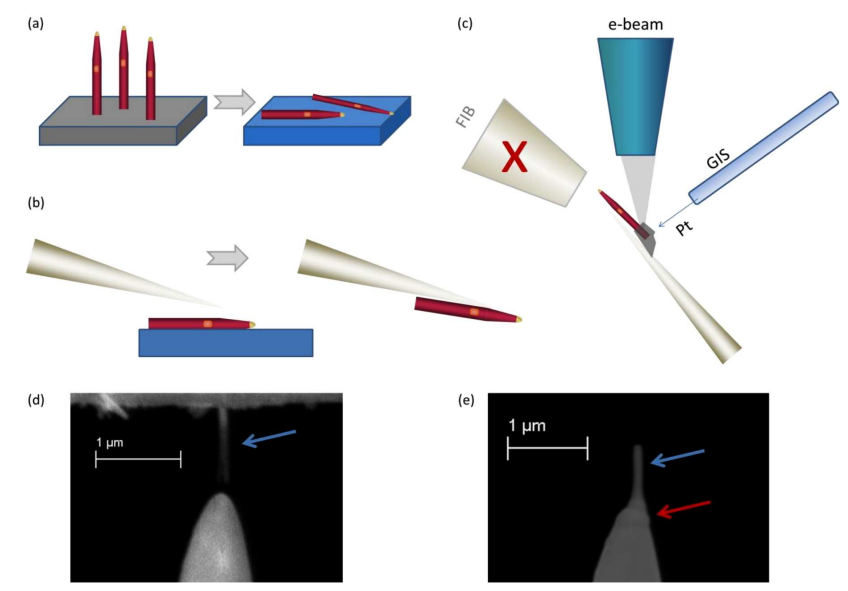

Fig. 9. Scheme of a FIB-free nanowire preparation protocol for correlated optical-APT study: (a) nanowire transfer from the growth substrate to a host substrate; (b) nanowire collection by micromanipulation under an optical or scanning electron microscope; (c) nanowire soldering by electron beam-induced deposition of a metal injected by a gas injection system (GIS). (d) SEM image of a $\mathrm{W}$ tip approaching an ultrashort GaN/AlGaN heterostructured nanowire for collection and (e) the nanowire collected and soldered by EBID. The blue and the red arrow indicate the nanowire and the Pt soldering, respectively.

the use of conductive epoxy, by the use of electron beamcurable glue, or simply by electrostatic forces. The two latter cases need a further step inside a SEM, either for curing the glue or for soldering the wire by electron beaminduced deposition (EBID) of metal, as shown in Fig. 9c, and are obviously possible when the collection step has been performed inside the SEM itself.

The example of the SEM-based collection and EBID soldering of an extremely small (700 nm length, $70 \mathrm{~nm}$ diameter) nanowire containing an $\mathrm{AlGaN} / \mathrm{GaN}$ axial multiple quantum disc system is illustrated in Fig. 9d,e. For collecting this wire, the tungsten support tip is directed by micromanipulation towards the edge of the growth substrate of the nanowires. Isolated nanowires may be found on the edge of the substrate, where they acquire a convenient orientation. In this particular case, the collection and soldering steps have been performed simultaneously, as the nanowire did not stick by electrostatic forces to the $\mathrm{W}$ tip just upon simple mechanical contact.

3.2.5. A correlative study of In $G a N / G a N$ nanowire quantum wells

This section illustrates an example of correlated optical-structural study of the same nanoscale object by $\mu \mathrm{PL}$ and APT. For sake of completeness, it should be mentioned that the nanoscale object was prepared with the two-step FIB-based protocol presented in Sect. 3.2.4, and that therefore future studies could still increase the accuracy of the correlation. This qualifies the study as an important step towards a "strictly correlative" approach. It also should be mentioned that the presented nanoscale 
object has undergone a further correlation with also HRSTEM, this analysis being performed between $\mu \mathrm{PL}$ spectroscopy and APT. Data relative to HR-STEM will also be presented, in order to clarify the specific contribution of this technique to the overall study. Further details can be found in Ref. [34] and in its supplementary information.

Micro-photoluminescence. The $\mu \mathrm{PL}$ study of the radial MQW system is described in Fig. 10. The microwires studied were grown by MOCVD. The growth is performed so that a thinner core is synthesized, then the growth conditions are changed in order to realize a conformal growth on the upper part of it. During conformal growth, a set of 20 QWs is inserted by modulating the $\mathrm{Ga}$ and In precursor fluxes. The external appearance of the wire is displayed in the SEM image of Fig. 10a, while Fig. 10b reports a scheme illustrating the quantum well arrangement. From this batch of wires, different micro- and nanoscale samples were prepared for $\mu \mathrm{PL}$ spectroscopy. Figure $10 \mathrm{~d}$ reports the $\mu \mathrm{PL}$ spectra recorded under $244 \mathrm{~nm} \mathrm{cw}$ laser excitation at $4 \mathrm{~K}$. The spectra displayed in the upper part of (a)

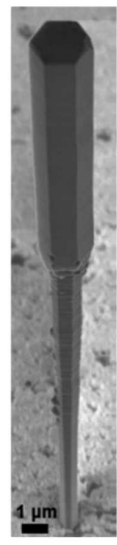

(b)

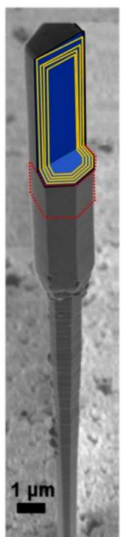

(c)

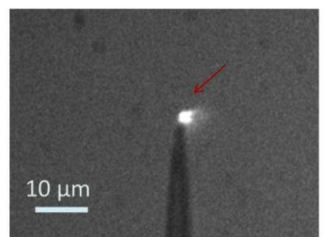

(d)
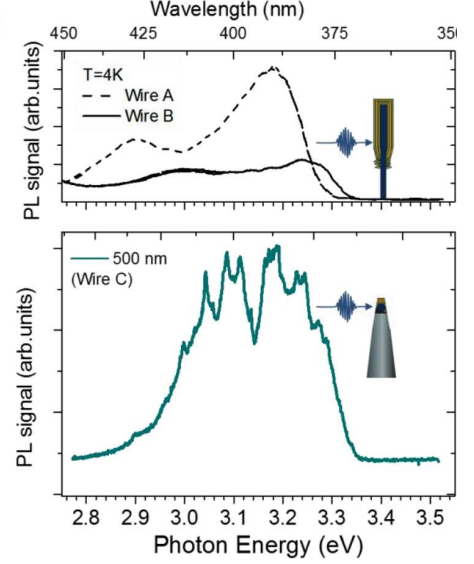

(e)

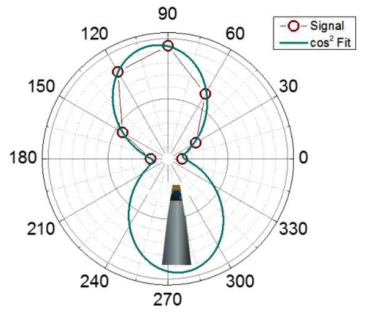

Fig. 10. (a) SEM image of one of the analyzed microwires; (b) scheme of the multishell quantum well system, with the red dashed line approximately indicating the region from which nanochunk $\mathrm{C}$ was extracted; (c) optical microscopic image of the PL (highlighted by the red arrow) emitted by nanochunk $\mathrm{C}$ mounted on a tungsten tip under excitation with a $244 \mathrm{~nm} \mathrm{cw}$ laser at $4 \mathrm{~K}$. (d) $\mu \mathrm{PL}$ spectra of two whole nanowires $\mathrm{A}$ and $\mathrm{B}$ mounted on a tungsten tip (black lines, upper part of the graph) and of nanochunk B which have been subsequently analyzed by APT; (e) polarization of the integral PL signal - the radial scale spans from 0 to 1 in normalized units. The plot has the same orientation as the image in part (c) (adapted with permission from Ref. [34]). the graph were collected from whole wires, while those in the lower part were issued by the nanochunk $\mathrm{C}$, which also appears in the left-hand side SEM image of Fig. 8b. The PL spot emitted by nanochunk $\mathrm{C}$ is also visualized in the microscopic image of Fig. 10c. Despite the laser beam is tightly focused to a spot of $\approx 1 \mu \mathrm{m}$ size, illuminating a specific region of the wire, the PL spectra of the whole wires consist of a large band, showing two main components but no particular fine structure. The situation is different when analyzing nanochunks. Here narrow lines appear, distributed in the interval 2.9-3.3 eV: this points out that a set of emitters with well-defined energy is contained in the nanochunk. The difference between the spectra of the whole wires and those of nanochunks depends on the probed volume: even if tightly focused, the laser still illuminates a much larger microwire volume when impinging on whole wires than when it illuminates the nanoscale chunks. Finally, $\mu \mathrm{PL}$ also allows for measuring the polarization of the emitted light, which is shown in the plot of Fig. 10e: the polarization is strongly parallel to the tip axis, with a quite high polarization rate

$$
P=\left(I_{\|}-I_{\perp}\right) /\left(I_{\|}+I_{\perp}\right)=0.89,
$$

where $I_{\|}$and $I_{\perp}$ are the integral PL intensities polarized parallel and perpendicularly to the nanochunk axis, respectively. As the nanochunk has a relatively large diameter, the polarization is rather expected to be determined by intrinsic crystalline selection rules than by the dielectric contrast between the object and the surrounding environment [35]. On the other hand, the polarization direction found agrees rather well with the nominal orientation of the crystal within the nanochunk: the polarization along the $c$ wurtzite axis, perpendicular to the nanochunk and to the direction of the emitted light, is strictly forbidden according to the selection rules for the fundamental radiative transition in GaN and in low In-containing quantum wells, the so-called $\mathrm{X}_{\mathrm{A}}$ exciton (related to the recombination of a conduction band electron and a hole from the heavy hole band) [36]. The crystalline orientation of the chunk could be verified by TEM.

Transmission electron microscopy. After nanochunk C was further milled down as shown in Fig. 8c, it was analyzed by HR-STEM. The high-annular dark field (HAADF) images are shown in Fig. 11a,b. In part (a), the upper part or the specimen tip is imaged. The nearly horizontal bright contrast lines correspond to the InGaN QWs. 14 QWs are imaged, while the remaining 6 are left unobserved in order to control whether the electron beam could have induced a re-arrangement of the In atoms, as it was pointed out by Smeeton et al. [37]. This check has been performed a posteriori by APT, indicating that e-beam exposure was short enough to prevent artificial In clustering. Only QW \#4 has been observed at high resolution, in order to display the crystal symmetry and to perform a first analysis of the QW interfaces, as shown in the close-up of Fig. 11b. 
(a)

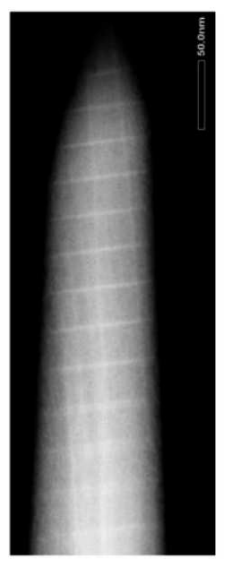

(b)

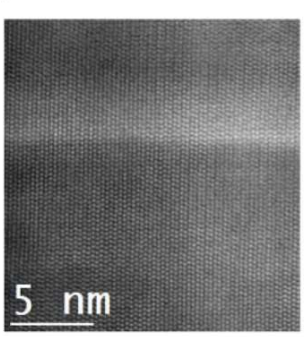

(c)

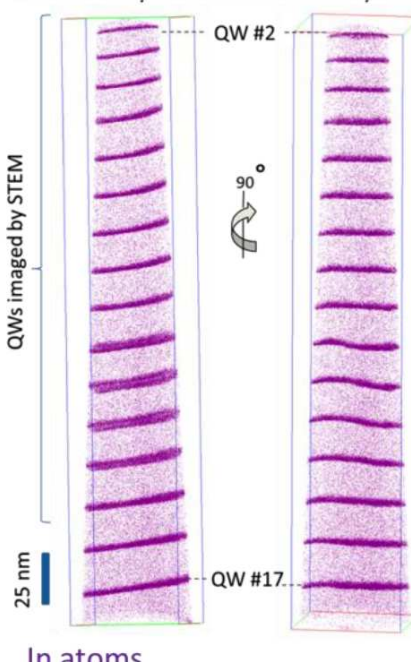

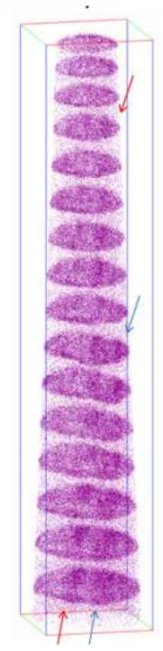

(e)

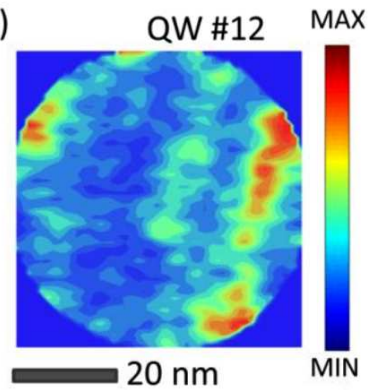

(d)

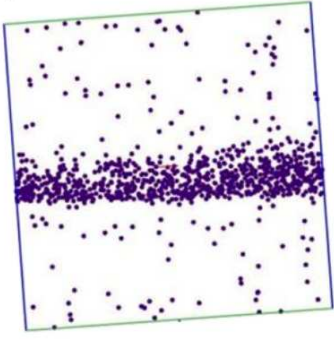

(f)

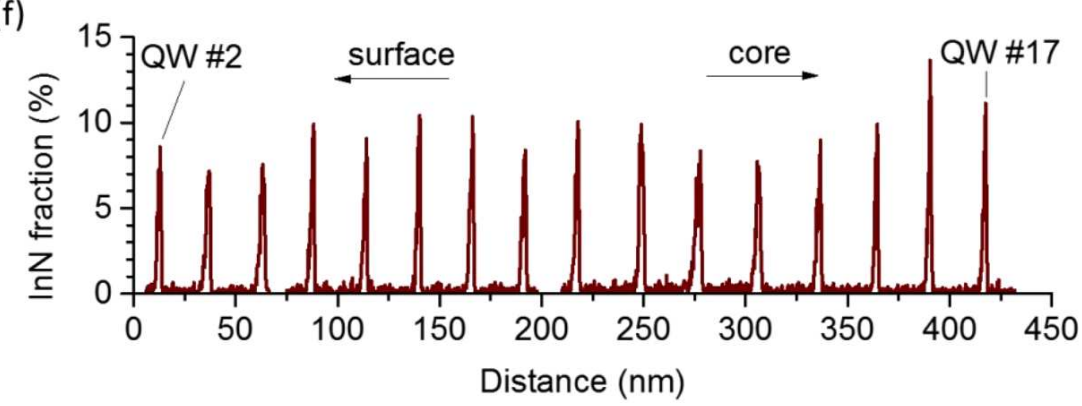

Fig. 11. (a) HAADF-STEM image of the tip milled out of nanochunk C: the bright contrast nearly horizontal lines correspond to InGaN quantum wells inside a GaN matrix; (b) close-up of QW \#4, showing the hexagonal wurtzite structure and its orientation: the m-direction [4120] is vertical on the image, perpendicular to the QW planes, while the c-direction [0001] lies horizontally on the image. (c) APT 3D reconstruction of 16 out of 20 QWs contained in the tip. Only $10 \%$ In atoms are shown for clarity; (d) close-up of a region of QW \#4; (e) 2D density plot of the InN fraction calculated in a rectangular box containing QW \#12; (f) $1 \mathrm{D}$ profile of the InN fraction obtained through the analysis of boxes with their z-axis perpendicular to the QW interfaces. The box surface parallel to the QW plane is $(10 \mathrm{~nm})^{2}$ (adapted with permission from Ref. [34]).

The STEM analysis confirms the nominal microwire crystal structure, with the wurtzite $c$ axis along the microwire axis and the QW planes aligned with the nonpolar $m$-planes. This confirmation of the crystal orientation, which is not retrievable by APT only, is important in connection with the optical properties as (i) it allows simulating the structures along the proper crystal direction: the $m$-type interfaces of these QWs are non-polar, while $c$-type interfaces would be polar, with a strong QCSE affecting the energy levels inside the QW, and (ii) it confirms that the PL polarization is related to the selection rules for the WZ low-In content QWs. The close-up of QW \#4 also shows that the interfaces of the QW are quite sharp on the core side, and somehow diffuse on the surface side. This feature should be further analyzed by APT. The image in Fig. 11a also displays nearly vertical bright contrast lines. Complementary analyses performed on other tips and on a lamellar specimen (not shown here) indicate quite clearly that these vertical lines are related to stacking faults 
(SFs) [34]. Furthermore, evidence for plastic relaxation via the formation of dislocations within the system was found. These complementary observations suggest that the QW system is at least partially relaxed, which is another important information for the interpretation of optical properties: in fact, the PL energy can also be influenced by the presence of stacking faults [38] and strain [36], and this information is not to be assessed by APT only.

Atom probe tomography. Finally, a large amount of information about the multi-QW system under study could be obtained by APT. The run was performed in a Lawatap, at temperature $T=20 \mathrm{~K}$, with an impinging laser energy per unit surface $5-10 \mu \mathrm{J} / \mathrm{cm}^{2}$ and a $\mathrm{Ga}^{2+} / \mathrm{Ga}^{1+}$ charge state ratio varying from 0.18 to 0.06 throughout the analysis, with a total of $33 \times 10^{6}$ ions detected. Figure 11c displays the position of $10 \%$ In atoms in the $3 \mathrm{D}$ reconstruction. The leftmost reconstruction image is oriented so to show the correspondence with the STEM image of part (a). A total of 16 QWs over 20 could be imaged by APT. The STEM image was profitably used to achieve the best possible reconstruction. Remarkably, a good reconstruction could only be obtained by postulating very low detection efficiency, of the order of 0.3 . This means that, the maximum detector efficiency being of the order of 0.66 , about one detectable atom over 2 was lost, most likely through a combination of uncorrelated evaporation (rather concerning $\mathrm{Ga}$ ) and neutral evaporation (rather concerning N). Further details about the III/ $\mathrm{N}$ compositional measurement and about the efficiency issue in reconstruction can be found in Ref. [39] and in its supplementary information, respectively.

The comparison between STEM and APT shows some interesting features: the apparently thicker QWs found in the particular zone axis adopted for the STEM imaging - we remind that we could not perform electron tomography, as for this technique the exposure times are necessarily very long, and we wanted to avoid long exposures to the e-beam - appear now as bent QWs in the APT reconstruction, with QWs as thick as the surrounding ones. Furthermore, there In distribution within the QW planes is not uniform: In-rich stripes directed approximately along the $c$-axis appear, with correlated positions along the different QW, as if they propagated along the $m$-axis. Figure 11d shows a close-up of a cross-section of QW \#4. This image should be compared with the HRSTEM image of Fig. 11b. Both images are consistent in indicating that the QW interface on the core side is better defined than that on the surface side. This is due to the growth method, in which the InGaN QW is grown at lower temperature $\left(T=750^{\circ} \mathrm{C}\right)$ than the GaN barriers $\left(T=870^{\circ} \mathrm{C}\right)[40,41]$. The InGaN at low temperature has a worse surface diffusion than GaN at high temperature, forming a rougher interface. Subsequently, GaN at higher temperature recovers a planar growth due to enhanced surface diffusion. The QWs are thus asymmetric, and this also has an effect on the electronic band structure. The asymmetry of the QW composition can be first visualized by plotting $1 \mathrm{D}$ profiles, as in Fig. 11e: these profiles are issued by averaging the composition over the cross- section of boxes of $(10 \mathrm{~nm})^{2}$ area, and show that all QWs have a FWHM thickness of around $3 \mathrm{~nm}$, with peak InN fractions fluctuating between $8 \%$ and $13 \%$.

Another key observation for the correlation of optical and structural properties is that the In in-plane distribution within QWs is far than uniform. This can be qualitatively visualized either by plotting the 3D distribution of the In atoms, as in Fig. 11b or by calculating the $2 \mathrm{D} \mathrm{In} /(\mathrm{In}+\mathrm{Ga})$ concentration averaged over the thickness of a rectangular box containing the whole QW. The actual InN fraction can be then correctly measured in 3D. This is reported for QW\# 12, but similar conclusions apply to the other QWs. The analysis was performed by isolating $1 \mathrm{~nm}$ thick slices within the well, approximately parallel to the $m$-plane, as shown in Fig. 12a and calculating the $\mathrm{InN}$ fraction for each $2 \mathrm{D}$ slice. The result is displayed in Fig. 12b. The measurements indicate that the peak InN fraction is around $20 \%$, which is much larger than what could be obtained by $1 \mathrm{D}$ profiling due to averaging effects between high- and low-In content regions. We also remark that the distribution of In in the QWs not illuminated by the electron beam in the STEM but imaged by APT is similar to the QWs imaged by STEM, indicating that the exposure to the electron beam was sufficiently short not to induce an artificial In rearrangement within the system [42].

Summary: what can be extracted from a triple correlated analysis on the same nano-object. All information described above allows thus for a consistent interpretation of the relationship between structural and optical properties of the nanoscale object analyzed. The main conclusions could be drawn by considering the plot in Fig. 12c, which reports on the ordinates the PL emission energy (with the $\mu \mathrm{PL}$ spectrum of nanochunk $\mathrm{C}$ superimposed) and on the abscissa the InN composition. The different curves represent the calculation of the PL energy of interband transitions taking place in relaxed $m$-plane non-polar rectangular QWs of different thickness as a function of the InN fraction. The bandgap of bulk $\mathrm{InGaN}$ is also reported as a reference. In the plot there are also highlighted the InN fractions found by $1 \mathrm{D}$ profiling (up to $13 \%$ ) of APT data and by 3D measurement (up to $20 \%$ ). The conclusions that could be drawn on this basis are the following:

- The multiple peaks correspond to regions where InN segregation creates local potential wells in which both electrons and holes localize, in a quantum dot-like confining system. The dispersion in the peak energies correspond to the different In content in these regions.

- The 3D measurement of InN fraction can account for all recorded PL energies, while the lowest energies could not be explained by a simpler 1D profiling.

Furthermore, other conclusions concerning the optical properties can be drawn (some of them are repeated here 
as a summary) on the basis of the correlation with the TEM data:

- The QWs are non-polar, and are at least partially relaxed. This information, used for the calculation of the transition energies, is consistent with the optical properties and with the APT data.

- The PL is polarized according to the selection rules for the electron-heavy hole transition in WZ GaN and in low InN-containing QWs.

Finally, we mention the information that could be assessed unambiguously thanks to the correlation between STEM and APT:

- The QWs are asymmetric due to the reduced InGaN surface diffusion during the growth of the QWs at the temperature of $750^{\circ} \mathrm{C}$.

- The best APT reconstruction could be obtained by postulating a detector efficiency of 0.3 only, pointing out that around one detectable atom over two is lost during APT analysis.

- The presence of bent QWs indicates that some accident could have perturbed their growth. This particular QW shape could not be assessed by simple STEM analysis observing along only one zone axis (this problem could have been overcome by electron tomography, but this technique requires long exposures, which were not desirable in the present case).

- The presence of stacking faults (SFs) crossing the quantum wells is expected to increase the localization of carriers at the intersection between the defect and the quantum well, with localization energies of the order of $100 \mathrm{meV}$ [38]. The consideration of this effect yields an even better agreement between the observed PL spectrum and the structural data obtained by APT and STEM.

\subsection{Beyond the correlative approach: the coupled, "in situ" approach}

The scheme of the principle of a coupled microphotoluminescence and tomographic atom probe instrument is sketched in Fig. 13. The basic idea is to exploit the femtosecond laser pulse in order to trigger the evaporation of single ions from a field emission tip placed in an intense electric field and, at the same time, to generate electron-hole pairs which can recombine radiatively yielding a luminescence signal. The fs laser pulse is focused onto the sample tip. The evaporated ions are collected on the $2 \mathrm{D}$ position and time-of-flight sensitive detector. Their $\mathrm{X}$ and $\mathrm{Y}$ positions, the time of flight tof and the event number in the evaporation sequence are recorded. These data are then elaborated in order to yield the 3D sample reconstruction, mass spectra, and time-of-flight spectra, as in a standard atom probe experiment. It must be noticed here that most atom probe (a)

QW \#12
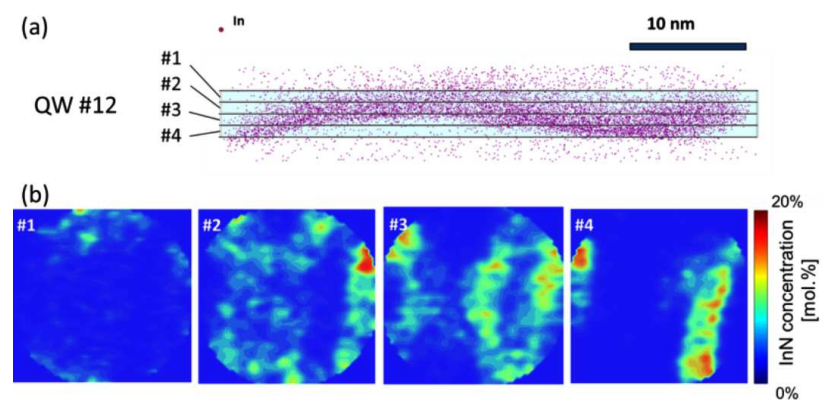

(c)

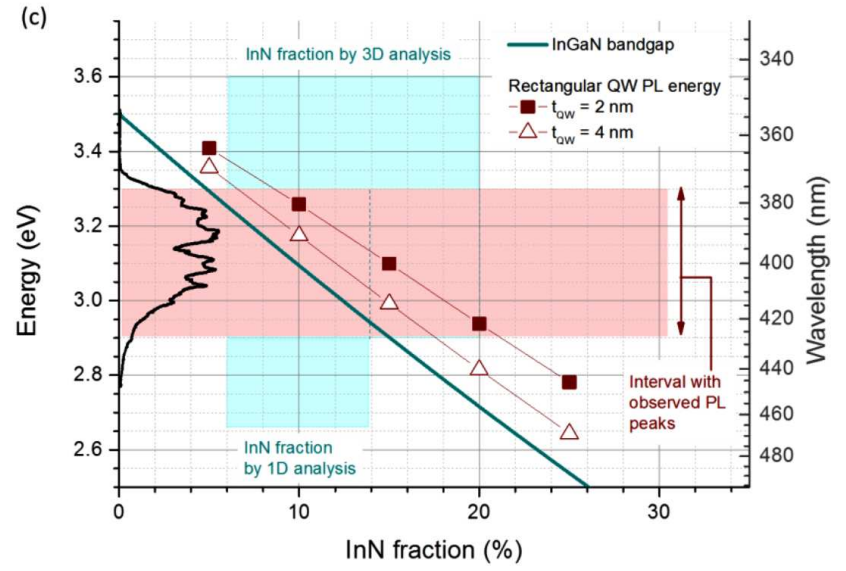

Fig. 12. (a) Close-up of the 3D distribution of QW \#12, showing the $1 \mathrm{~nm}$ thick slices for the calculation of the InN fraction maps shown in (b). (c) Dependence on InN fraction of the InGaN bandgap (blue line) and of the InGaN/GaN PL transition energies calculated for rectangular quantum wells of different thickness $t_{\mathrm{QW}}=2 \mathrm{~nm}$ (filled squares), and $t_{\mathrm{QW}}=4 \mathrm{~nm}$ (open triangles). The red shaded region corresponds to the interval in which the PL narrow lines have been observed in the analyzed nanochunk from wire $\mathrm{C}$; the $\mu \mathrm{PL}$ spectrum is also reported on the left-hand side axis (black line) (adapted with permission from Ref. [38]).

experiments are performed keeping the sample base at a temperature varying between $20 \mathrm{~K}$ and $100 \mathrm{~K}$. This is also a favorable interval for the analysis of the PL signal, as radiative recombination is favored at low temperature due to the freeze-out of non-radiative channels. A fraction of the emitted photons, depending on the sample geometry and optoelectronic properties, is collected by the same objective and focused on the entry slit of a spectrometer provided with a spectrally and time-resolved detector, such as a streak camera. It becomes thus possible to analyze the time decay of the PL signal in a selected spectral interval, whose resolution and width may be adjusted through the settings of the spectrometer.

A coupled optical spectroscopy and atom probe tomography instrument would therefore be interesting for both the materials scientist and the atom probe developer because of the following:

- It would allow performing strict correlations between optics and structure in a more compact and straightforward way, minimizing the manipulation 


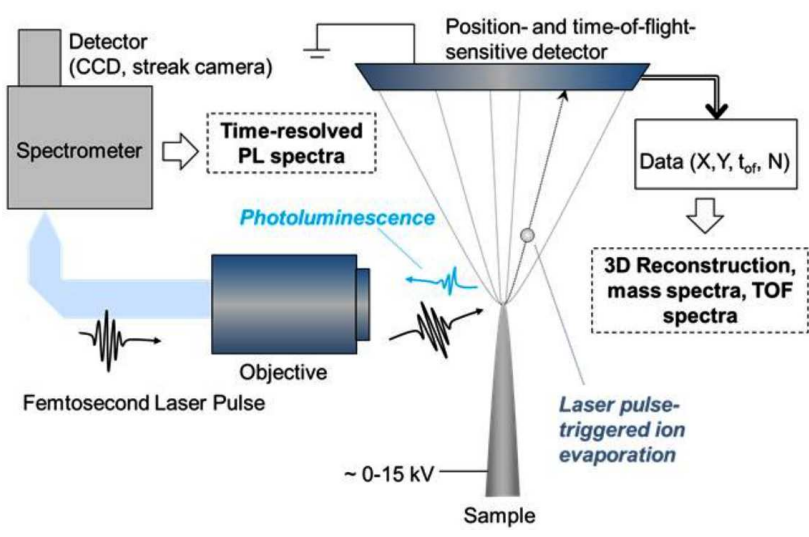

Fig. 13. Scheme of an in situ micro-photoluminescence bench inside a tomographic atom probe.

of the specimen between different experiments and decreasing the risk of specimen failure.

- It would allow for profiling the optical properties of the sample during the evaporation. It becomes thus possible to attribute spectral features to specific sample regions correlating their presence in the spectra with the evaporated portions of the specimen.

- It would be possible to study the QCSE in an original way, as the field penetrating within the tip is expected to influence the emission properties of localized quantum emitters such as quantum wells and dots. Furthermore, the measurement of the field effectively penetrating within the tip specimen is of particular interest for the understanding of field ion emission physics in dielectrics $[43,44]$.

However, it also should be mentioned that this approach also has the following limitations with respect to a simple correlative approach:

- It is not possible to perform a preliminary study by HR-STEM, as the electron energies used (> $100 \mathrm{kV}$ ), still preserving the structure of the systems, induce a deterioration of the optical properties, for instance strongly reducing the radiative recombination efficiency.

- The possibility of performing $\mu \mathrm{PL}$ and APT simultaneously is critically depending on the matching of experimental parameters. If it appears convenient to perform both analyses at low temperature and with a laser wavelength exceeding the specimen bandgap, further checks must be performed concerning the laser pulse energy and the applied DC bias.

\section{Acknowledgments}

The author acknowledges funding through projects EMC3 Labex ASAP and ANR-13-JS10-0001-01 Tapoter. He also thanks the organizers of the Jaszowiec 2015 conference for the kind invitation, and his collaborators from the Groupe de Physique des Matériaux (L. Mancini, D. Shinde, F. Vurpillot, A. Vella, W. Lefebvre, B. Deconihout, D. Blavette, I. Blum, D. Hernandez-Maldonado, J. Houard, M. Gilbert, E. Talbot, G. Da Costa, A. Bostel) for the fruitful discussions and for their contribution to the results illustrated in this work.

\section{References}

[1] B. Gault, M.P. Moody, J.P. Cairney, S.P. Ringer, Atom Probe Microscopy, Springer, New York 2012.

[2] M.K. Miller, R.G. Forbes, Atom-Probe Tomography: The Local Electrode Atom Probe, Springer: New York 2014.

[3] D.J. Larson, T.J. Prosa, R.M. Ulfig, B.P. Geiser, T.F. Kelly, Local electrode atom probe tomography, Springer, New York 2014.

[4] B. Gault, F. Vurpillot, A. Vella, M. Gilbert, A. Menand, D. Blavette, B. Deconihout, Rev. Sci. Instrum. 77, 043705 (2006).

[5] T. Kelly, M.K. Miller, Rev. Sci. Instrum. 78, 031101 (2007).

[6] T.F. Kelly, D.J. Larson, K. Thompson, R.L. Alvis, J.H. Bunton, J.D. Olson, B.P. Gorman, Ann. Rev. Mater. Sci. 37, 681 (2007).

[7] D. Blavette, S. Duguay, EPJ: Appl. Phys. 68, 10101 (2014).

[8] W.J. Choyke, G. Pensl, MRS Bull. 22, 25 (March 1997).

[9] M. Grundmann, The Physics of Semiconductors, Springer, Berlin 2010.

[10] A. Mascarenhas, S. Francoeur, S. Yoon, in: Dilute Nitride Semiconductors, Ed. M. Henini, Elsevier, Oxford 2005, p. 179

[11] A. Ziani, C. Davesnne, C. Labbé, J. Cardin, P. Marie, C. Frilay, X. Portier, Thin Solid Films 553, 52 (2014).

[12] J.H. Davies, The Physics of Low-Dimensional Semiconductors: An Introduction, Cambridge University Press, New York 1997.

[13] G. Bastard, Wave Mechanics Applied to Semiconductor Heterostructures, Les Editions de Physique, Paris 1988.

[14] P. Harrison, Quantum Wells, Wires and Dots: Theoretical and Computational Physics of Semiconductor Nanostructures, Wiley, Chichester 2005.

[15] B. Gil, Low-Dimensional Nitride Semiconductors, Oxford University Press, Oxford 2002.

[16] D.A.B. Miller, D.S. Chemla, T.C. Damen, A.C. Gossard, W. Wiegmann, T.H. Wood, C.A. Burrus, Phys Rev. Lett. 53, 2173 (1984).

[17] Y.H. Kuo, Y.K. Lee, Y. Ge, S. Ren, J.E. Roth, T.I. Kamins, J.S. Harris, Nature 437, 1334 (2005).

[18] F. Bernardini, V. Fiorentini, D. Vanderbilt, Phys. Rev. B 56, R10024 (1997).

[19] L.F. Zagonel, S. Mazzucco, M. Tencé, K. March, R. Bernard, B. Laslier, G. Jacopin, M. Tchernycheva, L. Rigutti, F.H. Julien, R. Songmuang, M. Kociak, Nano Lett. 11, 568 (2011). 
[20] Ł. Kłopotowski, V. Voliotis, A. Kudelski, A.I. Tartakovskii, P. Wojnar, K. Fronc, R. Grousson, O. Krebs, M.S. Skolnick, G. Karczewski, T. Wojtowicz, Phys. Rev. B 83, 155319 (2011).

[21] R. Kudrawiec, Phys. Status Solidi B 247, 1616 (2011).

[22] M. Heiss, Y. Fontana, A. Gustafsson, G. Wüst, C. Magen, D.D. O'Regan, A. Fontcuberta i Morral, Nature Mater. 12, 439 (2013).

[23] L. Mancini, Y. Fontana, S. Conesa-Boj, I. Blum, F. Vurpillot, L. Francaviglia, E. Russo-Averchi, M. Heiss, J. Arbiol, A. Fontcuberta i Morral, L. Rigutti, Appl. Phys. Lett. 105, 243106 (2014).

[24] S. Birner, Nextnano3 (accessed September 2014).

[25] L. Rigutti, G. Jacopin, L. Largeau, E. Galopin, A. De Luna Bugallo, F.H. Julien, J.C. Harmand, F. Glas, M. Tchernycheva, Phys. Rev. B 83, 155320 (2011).

[26] P. Billaud, S. Marhaba, E. Cottancin, L. Arnaud, G. Bachelier, C. Bonnet, N. Del Fatti, J. Lermé, F. Vallée, J.-L. Vialle, M. Broyer, M. Pellarin, J. Phys. Chem. C 112, 978 (2008).

[27] J.F. Ziegler, The Stopping and Range of Ions in Matter, srim.org, (accessed January 12, 2015).

[28] J. Mayer, L.A. Giannuzzi, T. Kamino, J. Michael, MRS Bull. 32, 400 (2007).

[29] L.F. Zagonel, L. Rigutti, M. Tchernycheva, G. Jacopin, R. Songmuang, M. Kociak, Nanotechnology 23, 455205 (2012).

[30] L. Rigutti, A. Vella, F. Vurpillot, A. Gaillard, N. Sevelin-Radiguet, J. Houard, A. Hideur, G. Martel, A. De Luna Bugallo, G. Jacopin, B. Deconihout, Ultramicroscopy 132, 75 (2013)

[31] M. Beversluis, A. Bouhelier, L. Novotny, Phys. Rev. B 68, 115433 (2003).

[32] A. Bouhelier, R. Bachelot, G. Lerondel, S. Kostcheev, P. Royer, G.P. Wiederrecht, Phys. Rev. Lett. 95, 267405 (2005).
[33] L. Rigutti, in: Wiley Encyclopedia of Electrical and Electronic Engineering, Wiley, 2015, p. 1.

[34] L. Rigutti, I. Blum, D. Shinde, D. HernándezMaldonado, W. Lefebvre, J. Houard, F. Vurpillot, A. Vella, M. Tchernycheva, C. Durand, J. Eymery, B. Deconihout, Nano Lett. 14, 107 (2014).

[35] H.E. Ruda, A. Shik, J. Appl. Phys. 100, 024314 (2006).

[36] S.L. Chuang, C.S. Chang, Phys. Rev. B 54, 2491 (1996).

[37] T.M. Smeeton, M.J. Kappers, J.S. Barnard, M.E. Vickers, C.J. Humphreys, Appl. Phys. Lett. 83, 5419 (2003).

[38] H. Jönen, U. Rossow, H. Bremers, L. Hoffmann, M. Brendel, A.D. Dräger, A. Hangleiter, Appl. Phys. Lett. 99, 011901 (2011).

[39] L. Mancini, N. Amirifar, D. Shinde, I. Blum, M. Gilbert, A. Vella, L. Rigutti, J. Phys. Chem. C 118, 24136 (2014).

[40] R. Köster, J.S. Hwang, C. Durand, D. Le Si Dang, J. Eymery, Nanotechnology 21, 015602 (2010).

[41] R. Köster, J.S. Hwang, D. Salomon, X. Chen, C. Bougerol, J.P. Barnes, D. Le Si Dang, L. Rigutti, A. De Luna Bugallo, G. Jacopin, M. Tchernycheva, C. Durand, J. Eymery, Nano Lett. 11, 4839 (2011).

[42] S.E. Bennett, D.W. Saxey, M.J. Kappers, J.S. Barnard, C.J. Humphreys, Appl. Phys. Lett. 99, 021906 (2011).

[43] E.P. Silaeva, M. Karahka, H.J. Kreuzer, Curr. Opin. Solid State Mater. Sci. 17, 211 (2013).

[44] E.P. Silaeva, L. Arnoldi, M.L. Karahka, B. Deconihout, A. Menand, H.J. Kreuzer, A. Vella, Nano Lett. 14, 6066 (2014). 